Document downloaded from:

http://hdl.handle.net/10251/122038

This paper must be cited as:

Navarro-Jiménez, J.; Tur Valiente, M.; Albelda Vitoria, J.; Ródenas, JJ. (2018). Large deformation frictional contact analysis with immersed boundary method. Computational Mechanics. 62(4):853-870. https://doi.org/10.1007/s00466-017-1533-x

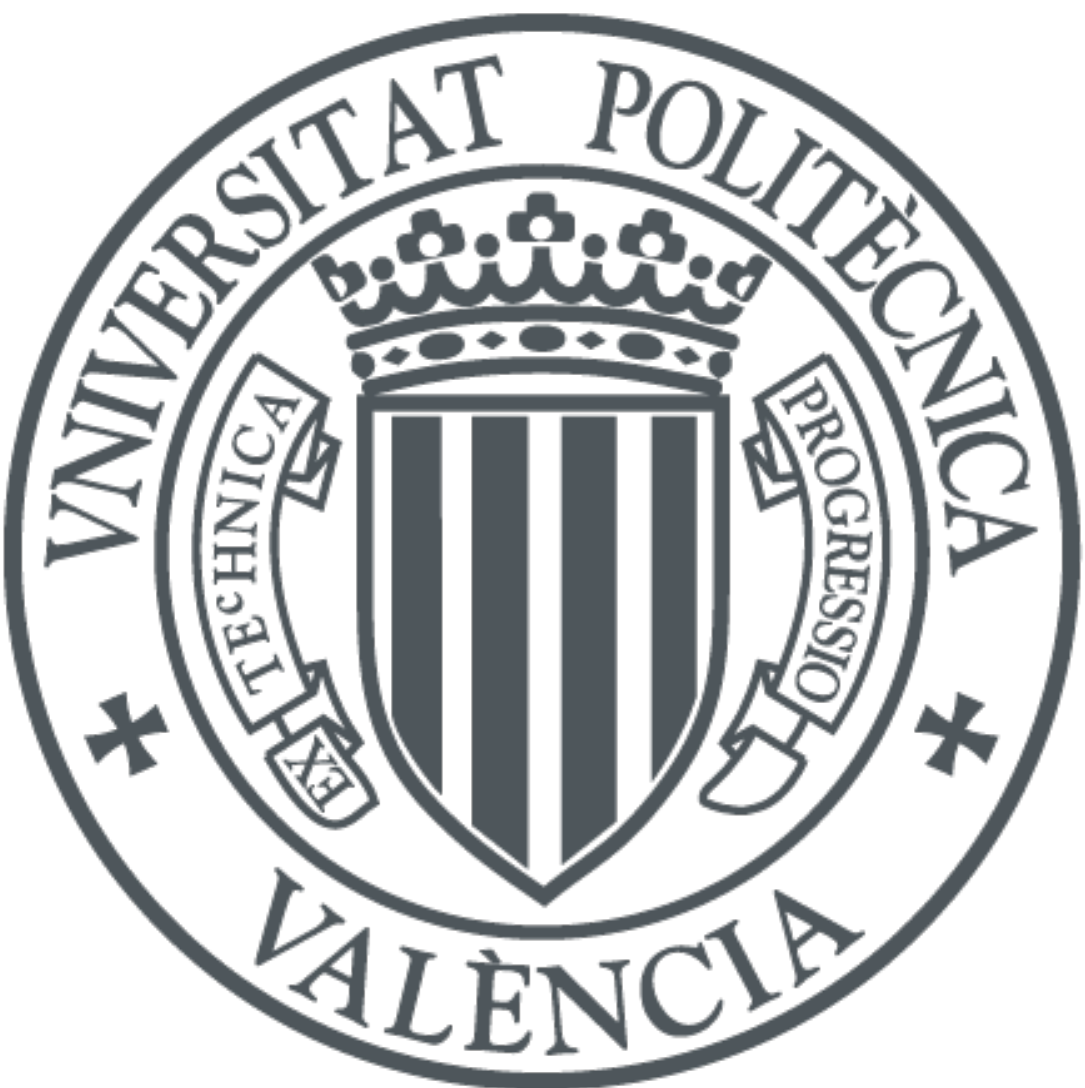

The final publication is available at

http://doi.org/10.1007/s00466-017-1533-x

Copyright Springer-Verlag

Additional Information 


\title{
Large deformation frictional contact analysis with immersed boundary method
}

\author{
José Manuel Navarro-Jiménez ${ }^{1}(\mathbb{D}) \cdot$ Manuel Tur ${ }^{1} \cdot$ José Albelda $^{1} \cdot$ Juan José Ródenas ${ }^{1}$
}

Received: 29 June 2017 / Accepted: 14 December 2017

c) Springer-Verlag GmbH Germany, part of Springer Nature 2017

\begin{abstract}
This paper proposes a method of solving 3D large deformation frictional contact problems with the Cartesian Grid Finite Element Method. A stabilized augmented Lagrangian contact formulation is developed using a smooth stress field as stabilizing term, calculated by Zienckiewicz and Zhu Superconvergent Patch Recovery. The parametric definition of the CAD surfaces (usually NURBS) is considered in the definition of the contact kinematics in order to obtain an enhanced measure of the contact gap. The numerical examples show the performance of the method.
\end{abstract}

Keywords Contact · Friction · Immersed boundary · Ficticious domain · cgFEM · Stabilized

\section{Introduction}

2 The so-called immersed boundary Finite Element (FE) methods have recently acquired notable relevance in the com4 putational mechanics field. The benefits of these methods include: virtually automatic domain discretization, suitability for efficient structural shape optimization and simplicity performing multigrid analysis. The present paper is based on the Cartesian grids Finite Element Method (cgFEM) [26], in which the domain is discretized by Cartesian grids independent of the geometry. The distinguishing feature of cgFEM is its ability to take into account the exact CAD definition of the geometry, given by NURBS. The development of a suitable contact formulation for the immersed boundary framework could be of interest for efficiently solving a number of different problems, e.g. wear simulation or fretting fatigue. In [15] the cgFEM is applied to directly create FE models from

\begin{tabular}{l}
\hline José Manuel Navarro-Jiménez \\
jonaji@upv.es \\
Manuel Tur \\
manuel.tur@mcm.upv.es \\
José Albelda \\
jalbelda@mcm.upv.es \\
Juan José Ródenas \\
jjrodena@mcm.upv.es \\
Centro de Investigación en Ingeniería Mecánica, \\
Departamento de Ingeniería Mecánica y de Materiales, \\
Universidad Politécnica de Valencia, Camino de Vera s/n, \\
46022 Valencia, Spain
\end{tabular}

medical images. The simulation of the contact interaction between CAD defined prostheses and living tissue, of great interest to the scientific community, can also be solved within this framework.

In this work a formulation for solving 3D frictional contact problems under large deformations is proposed, using an immersed boundary method based on Cartesian grids. The novelties of the present work are the use of a smooth stress field to iteratively evaluate the stabilizing term and the inclusion of the NURBS surface in the contact kinematics. The work presented in this paper represents an extension of a previous work [41], in which a stabilized formulation for solving frictionless contact problems was introduced and applied to body-fitted Finite Element meshes.

In the standard Finite Element Method (FEM) the mesh is conforming to the geometry. This means that the boundary is approximated by element faces defined from nodes lying on the boundary. Therefore, the geometry is approximated using the FE approximation (FE interpolation functions) used to define the solution. This provides a simple method of describing the domain in which the accuracy of the surface definition will depend on the level of refinement of the mesh. In this case the normal field is discontinuous between elements, which is an issue to consider when it comes to solving contact problems, as the measures of the gap between contact bodies are strongly influenced by the accuracy of the definition of the surfaces $[28,43]$. Some studies have tried to improve the quality of the contact kinematics description using various approaches, such as an averaged normal field $[34,46]$, the 
construction of smooth surfaces to evaluate the contact gap [28,43], and the application of the isogeometric analysis [22] to solve contact problems (see e.g. [10,11,39]). In this paper we include the NURBS surfaces in the contact kinematics to describe the reference configuration and enhance the accuracy of the gap measurements, while keeping the standard Finite Element interpolation for the solution of the problem.

The mortar method [9] has been used to successfully solve large deformation frictional contact problems [10,11,14,16, $34,39,42,46]$. Its main advantage over node-to-segment formulations is that the finite element optimal convergence rate of the solution is guaranteed, as the Brezzi-Babuska InfSup condition is fulfilled. However, the mortar method cannot be directly applied to deal with immersed boundary methods because it is cumbersome to find an appropriate Lagrange multipliers field that fulfills the InfSup condition [13]. The Vital Vertex method [7] can be used to find compatible displacement and stress fields, and was applied to $2 \mathrm{D}$ large sliding contact with XFEM in [29]. Other attempts to solve frictional contact using immersed boundary methods were in the context of simulating crack propagation with the eXtended Finite Element Method (X-FEM) [12,24,25,36]. Stabilized formulations are another alternative to overcoming this problem. Several works on this topic have been published, starting with stabilized Lagrange multipliers formulations for body-fitted meshes to solve small sliding 2D contact [21,35] and large deformation contact [30,33] in 2D and $3 \mathrm{D}$.

Stabilized formulations have been recently adapted to embedded domains. In [18] a stabilized augmented Lagrange formulation is developed for frictionless contact. A stabilized formulation based on the Nitsche method is presented in $[4,5]$ for small sliding contact in 2D and 3D respectively. In both formulations the stabilizing term involves the finite element tractions. All these contributions indicate that developing contact formulations for immersed boundary methods is an active research field. To the authors' knowledge no previous work has considered 3D CAD geometries and large deformation frictional contact for immersed boundary methods. A relevant difference between the proposed formulation and other works is its use of a smooth stress field $\left(\sigma^{*}\right)$ as stabilizing term, calculated by the Zienckiewicz and Zhu Superconvergent Patch Recovery [37,47]. With this choice there are fewer terms to evaluate in the tangent matrix, the formulation is displacement-based and the optimal convergence rate is maintained. It also eases the introduction of plasticity into the problem, as the finite element stress is not involved in the formulation (see [40]). The proposed formulation consists of two nested loops, similar to an Uzawa algorithm: the inner loop evaluates the contact active set and the stabilizing term is updated in an external loop.

The paper is organized as follows: Sect. 2 describes the continuum formulation to solve the contact problem. The contact kinematics and its features regarding the cgFEM is described in Sect. 3. The FE stabilized formulation is obtained in Sect. 4. In Sect. 5 we propose an iterative scheme to solve the contact problem. Finally, some numerical examples are shown in Sect. 6. Appendices A and B provide with details of the variation and linearization of some auxiliar terms of the problem formulation.

\section{Continuum formulation}

Here we describe the continuum formulation of the frictional contact problem and introduce all the notation used through the paper. The basic scheme of the contact between two elastic bodies, is shown in Fig. 1. We divide the boundary of each body $\Gamma^{(i)}$, into the Dirichlet boundary $\Gamma_{D}^{(i)}$, the Neumann boundary $\Gamma_{N}^{(i)}$ and the area of the boundary where contact may occur, $\Gamma_{C}^{(i)}$.

\subsection{Continuum contact kinematics}

Let $\mathbf{x}^{(1)}$ be the position of any point in the so called slave contact surface, $\Gamma_{C}^{(1)}$. We use a ray-tracing technique $[33,42]$ to define the contact point pairs, i.e. we intersect the master contact surface $\Gamma_{C}^{(2)}$ at $\mathbf{x}^{(2)}$ with a line emanating from $\mathbf{x}^{(1)}$ in the direction of the normal vector to the slave surface $\mathbf{n}^{(1)}$. Then the normal contact gap can be defined as

$g_{N}=\left(\mathbf{x}^{(2)}-\mathbf{x}^{(1)}\right) \cdot \mathbf{n}^{(1)}$

In order to enforce frictional contact constraints it is also necessary to define an appropriate relative velocity, from which the increment of the relative movement $\dot{\mathbf{g}} d t$ is obtained $[23,44]$ between the bodies in contact. Details of the calculation are not shown here, as it will be explained in Sect. 3.3 for the FE discretization using cgFEM.

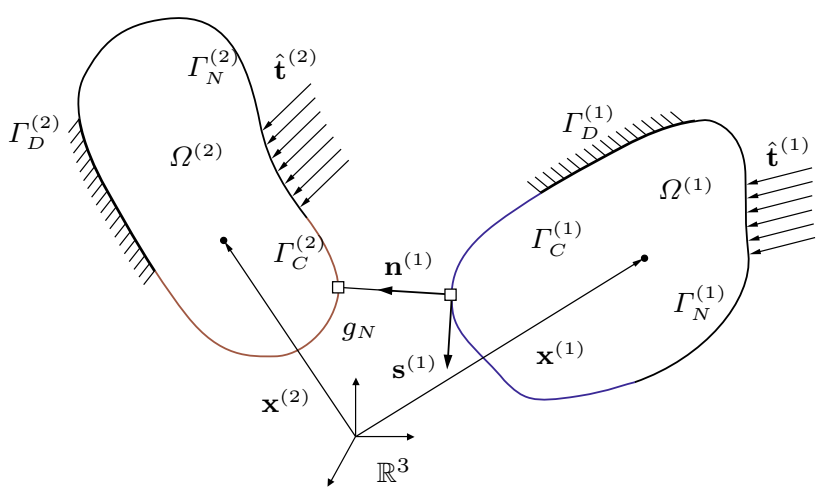

Fig. 1 Scheme of two deformable bodies in contact. The red and blue lines depict the contact boundaries $\Gamma^{(i)}$ 


\subsection{Weak formulation for frictional contact}

The weak formulation of the Tresca frictional problem can be derived from the augmented Lagrangian functional [33, 35], first proposed by Alart and Curnier [2] and Pietrzak and Curnier [32]:

$$
\begin{aligned}
& \text { opt }\left\{\Pi(\mathbf{u})+\frac{1}{2 \kappa_{1}} \int_{\Gamma_{C}^{(1)}}\left(\left[\lambda \cdot \mathbf{n}^{(1)}+\kappa_{1} g_{N}\right]_{-}^{2}-\|\lambda\|^{2}\right) d \Gamma\right. \\
& \left.+\frac{1}{2 \kappa_{1}} \int_{\Gamma_{C}^{(1)}}\left\|P_{B(n, s)}\left(\lambda-\kappa_{1} \dot{\mathbf{g}} d t\right)\right\|^{2} d \Gamma\right\}
\end{aligned}
$$

where $\mathbf{u}$ is the displacement field and $\lambda$ is the Lagrange multiplier vectorial field. We assume a hyperelastic material behavior so $\Pi(\mathbf{u})$ represents the potential energy of the bodies, including the external forces applied. $\kappa_{1}>0$ is a penalty constant that keeps the problem solution unchanged. We define the projection operator onto the tangent plane with normal $\mathbf{n}^{(1)}$ as:

$\mathbf{T}_{n}=\left(\mathbb{I}-\mathbf{n}^{(1)} \otimes \mathbf{n}^{(1)}\right)$

We also use the negative part operator

$[x]_{-}= \begin{cases}-x & \text { if } x \leq 0 \\ 0 & \text { if } x>0\end{cases}$

and the projection operator $P_{B(n, s)}(\mathbf{x})$ which is defined as the projection of $\mathbf{x}$ both on the tangent plane $\mathbf{T}_{n}$ and on a circle of radius $s$ :

$P_{B(n, s)}(\mathbf{x})= \begin{cases}\mathbf{T}_{n} \mathbf{x} & \text { if }\left\|\mathbf{T}_{n} \mathbf{x}\right\| \leq s \\ s \frac{\mathbf{T}_{n} \mathbf{x}}{\left\|\mathbf{T}_{n} \mathbf{x}\right\|} & \text { if }\left\|\mathbf{T}_{n} \mathbf{x}\right\|>s\end{cases}$

The stabilized Coulomb frictional contact formulation proposed in this work will be obtained by modifying the functional in 2. Taking variations in this equation and assuming a Tresca friction model (i.e. $s$ is constant) we obtain the following expression: of the internal and external forces, so the formulation in (6) can be applied to a general class of material behaviour. The contact integral in the first equation in (6) is the virtual work of the contact forces. The second equation contains the Karush-Kuhn-Tucker conditions in normal direction, and the frictional contact behaviour in the tangent plane. We can now modify the projection $P_{B}$ to consider Coulomb friction, i.e. replacing the friction limit $s$ with $\mu\left[\lambda_{N}+\kappa_{1} g_{N}\right]_{-}$, as done in [33].

After defining the weak form of the continuum problem, we replace the displacement and the Lagrange multiplier fields by appropriate finite element approximations, $\mathbf{u}^{h} \in$ $\mathscr{U}^{h}$ and $\lambda^{h} \in \mathscr{M}^{h}$, to obtain a numerical solution. $\mathscr{U}^{h}$ is the space of piecewise polynomials of degree $p=1$ or $p=2$ in our case. Details on the selection of the Lagrange multiplier approximation space are given in Sect. 4. For the sake of simplicity of the notation we will omit the superscript $h$ when denoting the finite element variables from now on.

\section{Finite element contact kinematics}

In this section we will define all the kinematic variables involved in the solution of the contact problem in the cgFEM, the normal contact gap $g_{N}$, the relative displacement $\mathbf{g} d t$ and the gap vector $\mathbf{g}$, and their respective variations.

In the cgFEM [26,27] the analysis domain $\Omega$ is fully embedded in a regular cuboid $\Omega_{h}$ which is much easier to mesh than $\Omega$, see Fig. 2 . This domain $\Omega_{h}$ is meshed with a sequence of regular Cartesian grids. There will be elements completely inside the domain and elements intersected by the boundary. The elements external to the domain are not considered in the analysis.

The geometry is defined by NURBS surfaces. Figure 3 shows the undeformed configuration of an element intersected by an arbitrary NURBS surface. Three different reference systems appear in the Figure: these are the global reference system $\mathbf{X}_{0} \equiv\left\{x_{0}, y_{0}, z_{0}\right\}$, the parametric reference system of the NURBS surface $\xi \equiv\{\xi, \eta\}$ and the local reference system of the finite element $\zeta^{e} \equiv\left[\zeta_{1}^{e}, \zeta_{2}^{e}, \zeta_{3}^{e}\right]$.

Due to the regularity of all the elements in the mesh, the transformation from global coordinates in the undeformed where the variations of $\mathbf{g}, \dot{\mathbf{g}} d t$, and $g_{N}$ are a function of $\delta \mathbf{u}$. The first term in the upper equation is the virtual work configuration $\mathbf{X}_{0}$ to element local coordinates $\boldsymbol{\zeta}^{e}$ of any point is performed with the following affine transformation: 

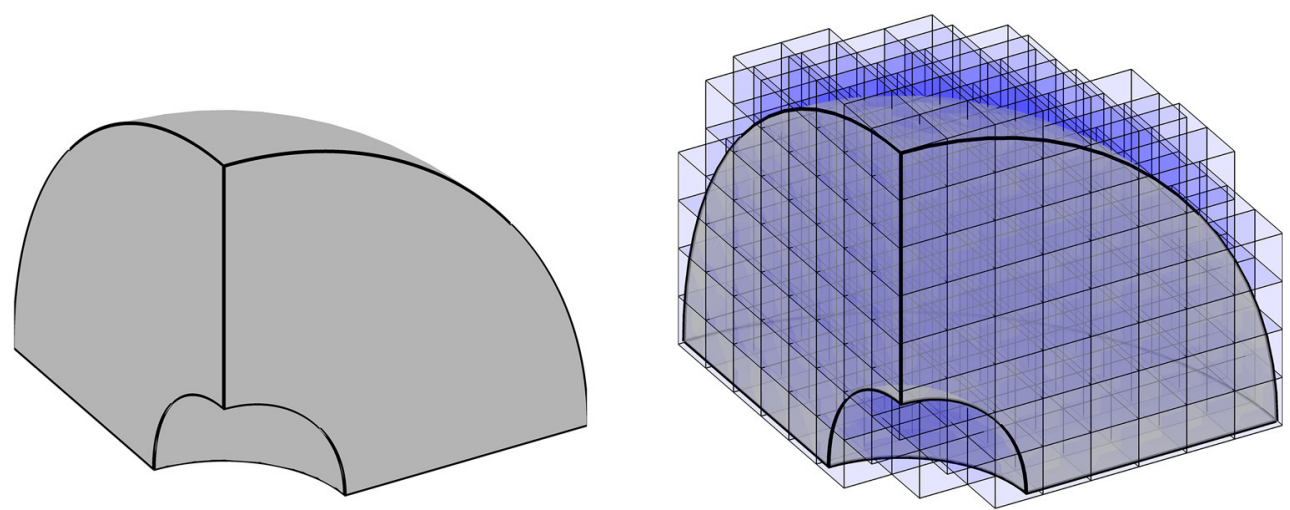

Fig. 2 Mesh creation with cgFEM. The analysis domain $\Omega$ (left) is embedded in a Cartesian grid $\Omega_{h}$ (right). Elements external to the geometry are not considered in the analysis

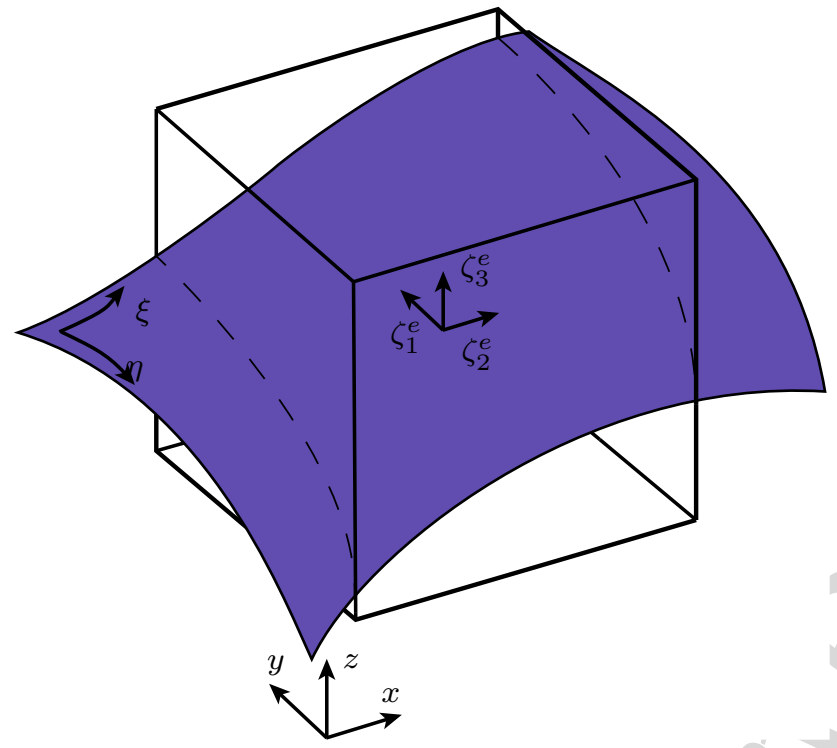

Fig. 3 Scheme of the different reference systems involved in the definition of the contact kinematics. The hexahedra represents a finite element cut by an arbitrary NURBS surface

197

${ }_{204} \mathbf{x}^{(i)}=\mathbf{X}_{0}^{(i)}+\mathbf{u}^{(i)}$
$\zeta^{e}=\frac{\mathbf{X}_{0}-\mathbf{X}_{e}}{h / 2}$

where $\mathbf{X}_{e}$ are global coordinates of the centroid of the element in the initial configuration and $h$ is the size of the element.

We define the position vector $\mathbf{x}^{(i)}$ for any point in $\Omega^{(i)}$ as in Eq. (8), where $\mathbf{X}_{0}^{(i)}$ represents the undeformed configuration and the displacement field $\mathbf{u}^{(i)}$ is evaluated using the finite element interpolation.

Equation (8) is valid for the whole domain, including the particular case of the contact surface, $\Gamma_{c}^{(i)}$. In this work we are interested in enhancing the definition of $\Gamma_{c}^{(i)}$ using the CAD geometry. We therefore use the NURBS definition of the boundary for the undeformed position for any point located at $\Gamma_{c}^{(i)}$. NURBS surfaces $[31,38]$ are rational functions defined in their own parametric space of coordinates $[\xi, \eta]$ as

$\mathbf{S}^{(i)}(\xi, \eta)=\sum_{i=1}^{n} \sum_{j=1}^{m} \frac{N_{i}^{(p)}(\xi) M_{j}^{(q)}(\eta) w_{i, j}}{\sum_{i=1}^{n} \sum_{j=1}^{m} N_{i}^{(p)}(\xi) M_{j}^{(q)}(\eta) w_{i, j}} \mathbf{P}_{i, j}$

These functions are a result of a tensor product between onedimensional basis functions of order $p$ and $q\left(N_{i}^{(p)}, M_{j}^{(q)}\right)$. The basis functions are defined along two knot vectors with $(n \times m)$ control points and $\mathbf{P}_{i, j}$ coordinates.

Finally, the definition of the position vector for any point $\mathbf{x}^{(i)}$ located at $\Gamma_{c}^{(i)}$ results in:

$\mathbf{x}^{(i)}=\mathbf{S}^{(i)}(\xi, \eta)+\sum_{j} N_{j}\left(\zeta^{e}\right) \mathbf{u}_{j}^{(i)}, \quad \mathbf{x}^{(i)} \in \Gamma_{c}^{(i)}$

where $N_{j}\left(\zeta^{e}\right)$ are the finite element shape functions and $\mathbf{u}_{j}^{(i)} \quad{ }_{220}$ are the nodal displacements of the discretization.

\subsection{Normal gap}

We recall here the definition of the normal gap $g_{N}$, where the position vectors have already been defined in (10):

$g_{N}=\left(\mathbf{x}^{(2)}\left(\xi^{(2)}\right)-\mathbf{x}^{(1)}\right) \cdot \mathbf{n}^{(1)}$

A ray-tracing technique is used to find the contact point $\xi^{(2)}$, i.e., given a certain point $\mathbf{x}^{(1)}$ and its surface normal vector $\mathbf{n}^{(1)}$, we solve (11), rearranged as:

$\mathbf{x}^{(1)}+g_{N} \mathbf{n}^{(1)}=\mathbf{S}^{(2)}\left(\boldsymbol{\xi}^{(2)}\right)+\sum_{j} N_{j}\left(\zeta^{e}\right) \mathbf{u}_{j}^{(2)}$
207 208 209 210 211

213 214 215 216 217 218 219 1 
${ }^{261} \delta g_{N}=\left(\delta \mathbf{u}^{(2)}-\delta \mathbf{u}^{(1)}\right) \cdot \mathbf{n}^{(1)}$ metric coordinates and the element local coordinates is obtained considering that for a point located on $\Gamma_{c}^{(i)}, \mathbf{X}_{0}^{(i)} \equiv$ $\mathbf{S}^{(i)}(\xi, \eta)$, and substituting (9) into (7)

$\zeta^{e}=\frac{\mathbf{S}^{(i)}(\xi, \eta)-\mathbf{X}_{e}}{h / 2}$

and taking derivatives with respect to the NURBS local coordinates $\xi \equiv\{\xi, \eta\}$ we obtain:

$\frac{\partial \boldsymbol{\zeta}^{e}}{\partial \xi}=\frac{2}{h} \frac{\partial \mathbf{S}^{(i)}(\xi, \eta)}{\partial \xi} ; \quad \frac{\partial \boldsymbol{\zeta}^{e}}{\partial \eta}=\frac{2}{h} \frac{\partial \mathbf{S}^{(i)}(\xi, \eta)}{\partial \eta}$

The calculation of the first derivatives of the NURBS follows a simple procedure (see [38] for example). The first derivatives have a similar definition to (9) with a lower order basis functions. Therefore the surface derivatives can be treated as auxiliary NURBS surfaces, and the evaluation of the NURBS derivatives is reduced to a standard NURBS surface evaluation.

The normal vector $\mathbf{n}^{(1)}$ is constructed using the tangent vectors to the surface, $\mathbf{s}_{\xi}^{(1)}$ and $\mathbf{s}_{\eta}^{(1)}$ (Eqs. (15), (16) and (17)).

$$
\begin{aligned}
\mathbf{n}^{(1)}= & \frac{\hat{\mathbf{n}}^{(1)}}{\left\|\hat{\mathbf{n}}^{(1)}\right\|} ; \quad \hat{\mathbf{n}}^{(1)}=\mathbf{s}_{\xi}^{(1)} \times \mathbf{s}_{\eta}^{(1)} \\
\mathbf{s}_{\xi}^{(1)}= & \frac{\partial \mathbf{x}^{(1)}}{\partial \xi}=\frac{\partial \mathbf{S}^{(i)}(\xi, \eta)}{\partial \xi} \\
& +\sum_{j}\left(\frac{\partial N_{j}}{\partial \zeta_{1}^{e}} \frac{\partial \zeta_{1}^{e}}{\partial \xi}+\frac{\partial N_{j}}{\partial \zeta_{2}^{e}} \frac{\partial \zeta_{2}^{e}}{\partial \xi}+\frac{\partial N_{j}}{\partial \zeta_{3}^{e}} \frac{\partial \zeta_{3}^{e}}{\partial \xi}\right) \mathbf{u}_{j}^{(1)}
\end{aligned}
$$

\subsection{Variation of the normal gap}

The contact problem formulation in (6) needs the definition of the normal gap variation. Instead of using the exact variation obtained from (11) we use an approximation which was also used in $[33,34]$, and can be written as where for simplicity the following notation has been introduced:

$\delta \mathbf{u}^{(i)}=\sum_{j} N_{j}\left(\zeta^{e}\right) \delta \mathbf{u}_{j}^{(i)}$

and $\mathbf{g}_{t+1}$ is normal to the tangent plane, so:

$\mathbf{T}_{n} \mathbf{x}_{t+1}^{(2)}\left(\xi_{t+1}\right)=\mathbf{T}_{n} \mathbf{x}_{t+1}^{(1)}$

With this consideration we can use the alternative definition of the projected relative velocity as:

$$
\begin{aligned}
\mathbf{T}_{n} \Delta^{t} \mathbf{g} & =\mathbf{T}_{n}\left(\mathbf{x}_{t+1}^{(2)}\left(\boldsymbol{\xi}_{t}\right)-\mathbf{x}_{t+1}^{(2)}\left(\boldsymbol{\xi}_{t+1}\right)\right) \\
& =\mathbf{T}_{n}\left(\mathbf{x}_{t+1}^{(1)}-\mathbf{x}_{t+1}^{(2)}\left(\boldsymbol{\xi}_{t}\right)\right)
\end{aligned}
$$




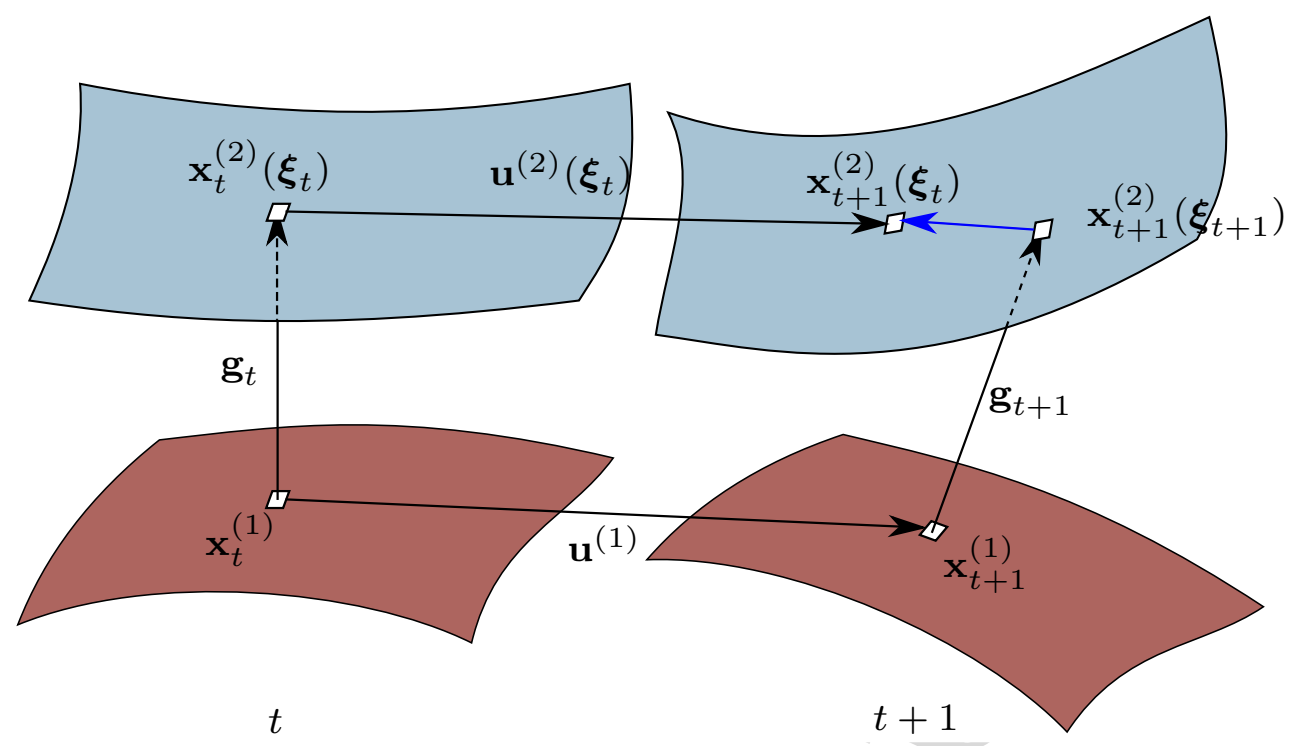

Fig. 4 Sliding kinematics scheme. In the configuration $t$, a point $\mathbf{x}_{t}^{(1)}$ is in contact with a point with local surface coordinates $\boldsymbol{\xi}_{t}$. After sliding occurs, the same point $\mathbf{x}_{t+1}^{(1)}$ will be contacting with a point $\mathbf{x}_{t+1}^{(2)}\left(\xi_{t+1}\right)$

This definition will provide us with a simpler linearization as it is shown in Appendix B. It is worth noting that, despite using the previous contact coordinates $\xi_{t}$ to evaluate the relative velocity, only the current configuration is taken into account. Note that in the case of sticking between the solids there is no change of the contact coordinates, then $\boldsymbol{\xi}_{t+1}=\boldsymbol{\xi}_{t}$ and we can combine the normal gap and the tangent relative velocity:

$g_{n} \mathbf{n}^{(1)}-\mathbf{T}_{n} \Delta^{t} \mathbf{g}=\mathbf{x}^{(2)}(\boldsymbol{\xi})-\mathbf{x}^{(1)}=\mathbf{g}$

This simplification will be useful for the stick contact formulation.

The variation of the gap vector is also used in the frictional contact formulation for the stick case, and defined with the simple expression:

$\delta \mathbf{g}=\delta \mathbf{u}^{(2)}(\boldsymbol{\xi})-\delta \mathbf{u}^{(1)}$

Again the derivatives $\delta \xi, \delta \eta$ will be omitted for the evaluation of the contact force, but will be considered for the linearization of the problem.

\section{Finite element stabilized contact formulation}

It is difficult to find a Lagrange multiplier field that fulfills the inf-sup condition in the immersed boundary framework [8]. The different methods of overcoming this problem include new formulations of the contact problem, such as modifications of the Nitsche method and stabilized Lagrangian formulations $[3,18,20]$. Here we extend the frictionless contact formulation first proposed in [41] to deal with frictional contact problems. Our proposed solution combines a stabilized augmented Lagrange formulation with the use of a smooth stress field $\mathbf{T}^{*}=\boldsymbol{\sigma}^{*} \cdot \mathbf{n}^{(1)}$ in the stabilizing term.

The smooth stress field used to stabilize the formulation must fulfill the following property $[19,40]$ in order to obtain an optimal FE formulation:

$\int_{\Gamma_{C}^{(1)}}\left\|\mathbf{T}^{*}\right\| \leq C \int_{\Omega}\left\|\boldsymbol{\sigma}^{*}\right\|^{2}$

with $\mathrm{C}$ independent of the mesh size. This condition states that the norm of the tractions on the boundary must be bounded by the norm of the stress field on the domain.

We use the field proposed in [42], which is based on the Zienckiewicz and Zhu Superconvergent Patch Recovery $[37,47]$. With this technique a smooth stress field is obtained by solving a small minimization problem at each node of the mesh. Once the displacements are known, the information of the solution at all the elements attached to the node is used to obtain $\sigma^{*}$. As the stabilizing term has information not only from the boundary elements but also from the surrounding interior elements, it can be proven that the optimal convergence rate for the FE solution is achieved, even if there are elements cut by the boundary with a low ratio between the intersected material volume and the whole element volume. This definition requires an iterative procedure to solve the problem, which will be detailed in Sect. 5 . 
We modify the augmented Lagrangian functional (2) with the addition of a stabilizing term [the last integral in (27)].

$$
\begin{aligned}
\text { opt } & \left\{\Pi(\mathbf{u})+\frac{1}{2 \kappa_{1}} \int_{\Gamma_{C}^{(1)}}\left(\left[\lambda \cdot \mathbf{n}^{(1)}+\kappa_{1} g_{N}\right]_{-}^{2}-\|\lambda\|^{2}\right) d \Gamma\right. \\
& ++\frac{1}{2 \kappa_{1}} \int_{\Gamma_{C}^{(1)}}\left\|P_{B}\left(\lambda-\kappa_{1} \Delta^{t} \mathbf{g}\right)\right\|^{2} d \Gamma \\
& \left.-\frac{1}{2 \kappa_{2}} \int_{\Gamma_{C}^{(1)}}\left\|\lambda-\mathbf{T}^{*}\right\|^{2} d \Gamma\right\}
\end{aligned}
$$

where the simplification $P_{B}(\mathbf{x}) \equiv P_{B\left(\mathbf{n}^{(1)}, \mu\left[\lambda_{N}+\kappa_{1} g_{N}\right]_{-}\right)}(\mathbf{x})$ is introduced. This extra term penalizes the difference between the multiplier $\lambda$ and the stress field using a penalty constant $\kappa_{2}>0$. In [40] the penalty constant is defined as $\kappa_{2}=C / h$ with $h$ being the mesh size and $C$ a positive constant. It was proved for Dirichlet boundary conditions that, for $C$ greater than a certain value depending only on the material properties and the degree of discretization, the problem is stable and the optimal convergence is reached.

Assuming that $\mathbf{T}^{*}$ is known, the variation of (27) is now written as: tinuous piecewise interpolation, we define a multiplier for each of the quadrature points used for the numerical integration. The Lagrange multipliers can the be condensed element by element as described in [41] (or even for every quadrature point), similar to the procedure followed in [6]. This elimination has some advantages: (a) the number of degrees of freedom of the problem does not increase, and (b) the system remains positive definite.

Remark The contact integrals over $\Gamma_{C}^{(1)}$ are numerically calculated on the integration points where the Lagrange multipliers are defined. This introduces an integration error, which is small if the number of integration points is high enough.

\subsection{Frictionless contact formulation}

The variational form for the Coulomb frictional contact in (28) can be simplified for the particular case of frictionless contact, yielding the following form:

$$
\left\{\begin{array}{l}
\delta \Pi(\mathbf{u}, \delta \mathbf{u})-\int_{\Gamma_{C}^{(1)}}\left(\left[\lambda \cdot \mathbf{n}^{(1)}+\kappa_{1} g_{N}\right]_{-} \delta g_{N}+P_{B}\left(\lambda-\kappa_{1} \Delta^{t} \mathbf{g}\right) \delta \mathbf{g}\right) d \Gamma=0, \quad \forall \delta \mathbf{u} \\
-\frac{1}{\kappa_{1}} \int_{\Gamma_{C}^{(1)}}\left(\left[\lambda \cdot \mathbf{n}^{(1)}+\kappa_{1} g_{N}\right]_{-} \mathbf{n}^{(1)}+\lambda-P_{B}\left(\lambda-\kappa_{1} \Delta^{t} \mathbf{g}\right)\right) \delta \lambda d \Gamma-\frac{1}{\kappa_{2}} \int_{\Gamma_{C}^{(1)}}\left(\lambda-\mathbf{T}^{*}\right) \delta \lambda d \Gamma=0, \quad \forall \delta \lambda
\end{array}\right.
$$

Remark In this paper we will enforce the contact constraint only on surface $\Gamma_{C}^{(1)}$ for the sake of simplicity. However, [41] shows how to use a double pass strategy to enforce the contact constraint on both surfaces $\Gamma_{C}^{(1)}$ and $\Gamma_{C}^{(2)}$ without additional complexity.

\subsection{Lagrange multiplier interpolation}

The requirements for the multiplier space to reach optimal convergence is that $\lambda^{h}$ be a piecewise interpolation in the element of degree at least $p-1$, where $p$ is the interpolation degree used to define $\mathbf{u}^{h}$. As there is no need to define a con- where we have introduced the normal stabilizing stress $p_{N}=$ $\left(\mathbf{T}^{*} \cdot \mathbf{n}^{(1)}\right) \cdot \mathbf{n}^{(1)}$. Taking into account the numerical integration, we have one equation for every quadrature point, depicted with the subindex $g$. Then, the following result can be obtained if we condense the Lagrange multipliers in the second equation in (29):

$\lambda_{N g}=\left\{\begin{array}{lll}\kappa_{2} g_{N g}+p_{N g} & \text { if } & {\left[\lambda_{N g}+\kappa_{1} g_{N g}\right]_{-} \leq 0} \\ 0 & \text { if }\left[\lambda_{N g}+\kappa_{1} g_{N g}\right]_{-}>0\end{array}\right.$

Substituting the Lagrange multiplier in (29) we will have the following equation to solve the normal contact problem. 


$$
\begin{aligned}
& \delta \Pi(\mathbf{u}, \delta \mathbf{u})-\sum_{g}\left(p_{N_{g}}+\frac{\kappa E}{h} g_{N_{g}}\right) \delta g_{N g} \\
& \left|J_{g}\right| H_{g}=0, \quad \text { if }\left[p_{N_{g}}+\frac{\kappa E}{h} g_{N g}\right]_{-} \leq 0 \\
& \delta \Pi(\mathbf{u}, \delta \mathbf{u})=0, \quad \text { if }\left[p_{N_{g}}+\frac{\kappa E}{h} g_{N_{g}}\right]_{-}>0
\end{aligned}
$$

where $H_{g}$ and $\left|J_{g}\right|$ are the respective quadrature weight and Jacobian of the transformation, and $\frac{\kappa E}{h}=\left(\kappa_{1}+\kappa_{2}\right)$ with $E$ being the Young's modulus and $h$ the mesh size. This result is similar to the one obtained in [18] with the advantage of having less integrals to evaluate as no derivatives of the stabilizing traction are involved in the formulation. Further discussion on the values of the stabilizing term can be found in [41].

\subsection{Frictional contact formulation}

Here we extend the stabilized formulation to the Coulomb frictional contact case with large deformations. We assume that the contact condition is active, i.e. $\left[p_{N}+\frac{\kappa E}{h} g_{N}\right]_{-} \leq 0$, otherwise the problem equation would remain as the second equation in (31). We can again substitute the value at the quadrature points of $\lambda_{N}$ obtained in (30), so that the Coulomb friction limit is written as $\mu\left[p_{N}+\frac{\kappa E}{h} g_{N}\right]_{-}$. It is also possible to condense element-wise the Lagrange multipliers using the second equation in (28). In order to do that, we will distinguish between the different states during frictional contact, the sticking case and the sliding case.

Starting with the stick state, we can substitute the corresponding value $P_{B}=\mathbf{T}_{n}\left(\lambda-\kappa_{1} \Delta^{t} \mathbf{g}\right)$ in the second equation in (28):

$-\frac{1}{\kappa_{1}}\left(\left[\lambda \cdot \mathbf{n}^{(1)}+\kappa_{1} g_{N}\right]_{-} \mathbf{n}^{(1)}+\lambda-\mathbf{T}_{n}\left(\lambda-\kappa_{1} \Delta^{t} \mathbf{g}\right)\right)$

$-\frac{1}{\kappa_{2}}\left(\lambda-\mathbf{T}^{*}\right)=0$

Hence, (32) can be simplified taking into account that $\lambda=$ $\left(\lambda \cdot \mathbf{n}^{(1)}\right) \mathbf{n}^{(1)}+\mathbf{T}_{n} \lambda$. Therefore, the Lagrange multiplier can be substituted at each integration point by:

$\lambda_{g}=\mathbf{T}_{g}^{*}+\kappa_{2}\left(g_{N_{g}} \mathbf{n}^{(1)}-\mathbf{T}_{n} \Delta^{t} \mathbf{g}_{g}\right)$

After substituting the value in the first equation of (28), and taking into account the simplification of (24) $\mathbf{g}=g_{N} \mathbf{n}^{(1)}$ $\mathbf{T}_{n} \Delta^{t} \mathbf{g}$ valid only for the stick case, the contact contribution to the problem in the case of stick is written as:

$\delta \Pi_{C_{S t}}(\mathbf{u}, \delta \mathbf{u})=\sum_{g}\left(\frac{\kappa E}{h} \mathbf{g}_{g}+\mathbf{T}_{g}^{*}\right) \cdot \delta \mathbf{g}_{g}\left|J_{g}\right| H_{g}$
The elimination of the Lagrange multipliers in the frictionless and stick cases allows the problem to be transformed into a modified penalty method, with the advantages mentioned above. However, the elimination of the multipliers for the sliding case is cumbersome, as in this case the second equation in (28) reads as:

$$
\begin{gathered}
-\frac{1}{\kappa_{1}}\left(\left[\lambda \cdot \mathbf{n}^{(1)}+\kappa_{1} g_{N}\right]_{-} \mathbf{n}^{(1)}+\lambda+\mu\left(p_{N}+\frac{\kappa E}{h} g_{N}\right) \frac{\mathbf{T}_{n}\left(\lambda-\kappa_{1} \Delta^{t} \mathbf{g}\right)}{\left\|\mathbf{T}_{n}\left(\lambda-\kappa_{1} \Delta^{t} \mathbf{g}\right)\right\|}\right) \\
-\frac{1}{\kappa_{2}}\left(\lambda-\mathbf{T}^{*}\right)=0
\end{gathered}
$$

We can project this equation on the normal direction $\mathbf{n}^{(1)}$ and the tangent plane $\mathbf{T}_{n}$. The first projection yields the the same equation that was discussed in the frictionless case (30). The projection on the tangent plane leads to the following equation:

$$
\begin{aligned}
& -\frac{1}{\kappa_{1}}\left(\mathbf{T}_{n} \lambda+\mu\left(p_{N}+\frac{\kappa E}{h} g_{N}\right) \frac{\mathbf{T}_{n}\left(\lambda-\kappa_{1} \Delta^{t} \mathbf{g}\right)}{\left\|\mathbf{T}_{n}\left(\lambda-\kappa_{1} \Delta^{t} \mathbf{g}\right)\right\|}\right) \\
& -\frac{1}{\kappa_{2}} \mathbf{T}_{n}\left(\lambda-\mathbf{T}^{*}\right)=0
\end{aligned}
$$

This is the slip condition that, roughly speaking, (neglecting the stabilizing term, $\lambda=\mathbf{T}^{*}$ ) forces the tangent projection of the multiplier to have a modulus $\mu\left(p_{N}+\frac{\kappa E}{h} g_{N}\right)$ and the direction of $\mathbf{T}_{n} \Delta^{t} \mathbf{g}$. The addition of the stabilization term, if $\mathbf{p}_{T}=\mathbf{T}_{n} \cdot \mathbf{T}^{*}$ is chosen in the direction of $\mathbf{T}_{n} \Delta^{t} \mathbf{g}$ and modulus $\mu p_{N}$, becomes again the same constraint, so the equation is redundant.

Only the direction of $\mathbf{T}_{n} \boldsymbol{\lambda}$ is involved in the first equation in (28). We formulate an alternative approach for the sliding problem that will lead to the same solution by modifying this equation. We consider that the direction of $\mathbf{T}_{n} \boldsymbol{\lambda}$ is the same as the direction of $\kappa_{2} \mathbf{T}_{n} \Delta^{t} \mathbf{g}+\mathbf{p}_{T}$, which also has the direction of $\mathbf{T}_{n} \Delta^{t} \mathbf{g}$ in the problem solution. In order to avoid convergence problems, the transition between stick and slip has to be continuous. This is achieved with the following substitution:

$\mathbf{T}_{n} \lambda=\kappa_{2} \mathbf{T}_{n} \Delta^{t} \mathbf{g}+\mathbf{p}_{T}$

Introducing this substitution into the first equation in (28) we obtain the final equation to solve the sliding problem:

$$
\begin{aligned}
& \delta \Pi_{C_{S l}}(\mathbf{u}, \delta \mathbf{u})=\sum_{g}\left[\left(\frac{\kappa E}{h} g_{N}+p_{N}\right) \delta g_{N}\right. \\
& \left.\quad-\mu\left(\frac{\kappa E}{h} g_{N}+p_{N}\right) \frac{\mathbf{p}_{T}-\frac{\kappa E}{h} \mathbf{T}_{n} \Delta^{t} \mathbf{g}}{\left\|\mathbf{p}_{T}-\frac{\kappa E}{h} \mathbf{T}_{n} \Delta^{t} \mathbf{g}\right\|} \cdot \delta \mathbf{g}\right]\left|J_{g}\right| H_{g}
\end{aligned}
$$


This approximation means the sliding problem can be formulated with a modified penalty method similar to those obtained for the frictionless and sticking cases. The numerical examples in Sect. 6 show that the convergence is still achieved.

The stabilizing smooth stress field $p_{N}$ and $\mathbf{p}_{T}$ are considered independent of the solution $\mathbf{u}$ in the linearization step. The values are iteratively updated in the problem solution as shown in the next Section.

\section{Problem solution}

The formulation obtained to solve the frictional contact problem can be summarized as:

$$
\left\{\begin{array}{l}
\text { if }\left[p_{N_{g}}+\frac{\kappa E}{h} g_{N_{g}}\right]_{-}>0 \\
\qquad \Pi(\mathbf{u}, \delta \mathbf{u})=0 \\
\text { otherwise } \\
\text { if }\left\|\mathbf{T}_{n}\left(\mathbf{T}_{g}^{*}+\frac{\kappa E}{h} \mathbf{g}_{g}\right)\right\| \leq \mu\left(p_{N_{g}}+\frac{\kappa E}{h} g_{N_{g}}\right) \\
\quad \delta \Pi(\mathbf{u}, \delta \mathbf{u})+\delta \Pi_{C_{S t}}(\mathbf{u}, \delta \mathbf{u})=0, \\
\text { if }\left\|\mathbf{T}_{n}\left(\mathbf{T}_{g}^{*}+\frac{\kappa E}{h} \mathbf{g}_{g}\right)\right\|>\mu\left(p_{N g}+\frac{\kappa E}{h} g_{N_{g}}\right) \\
\delta \Pi(\mathbf{u}, \delta \mathbf{u})+\delta \Pi_{C_{S l}}(\mathbf{u}, \delta \mathbf{u})=0
\end{array}\right.
$$

The first equation corresponds to the case of no active contact condition. The evaluation of $\delta \Pi_{C_{S t}}$ is found in (34), whereas $\delta \Pi_{C_{S l}}$ is defined in (38).

\subsection{Solution algorithm}

The choice of the stabilizing term $\mathbf{T}^{*}$ requires an iterative process to solve (39). The proposed procedure, first introduced in [41] is shown in Algorihm 1.

During the $N-R$ loop the contact status for each integration point on the contact boundary $\Gamma_{C}^{(1)}$ is evaluated. When any integration point becomes active, it is set to stick contact for its first iteration. After that, the slip condition is evaluated and the relative velocity is calculated for the sliding integration points.

An additional loop is needed for the solution of the problem to update the stabilizing stress field. Here it is called augmentation loop because of the similarities with the augmented Lagrange multipliers approach. Our experience shows that the number of augmentations is usually low,

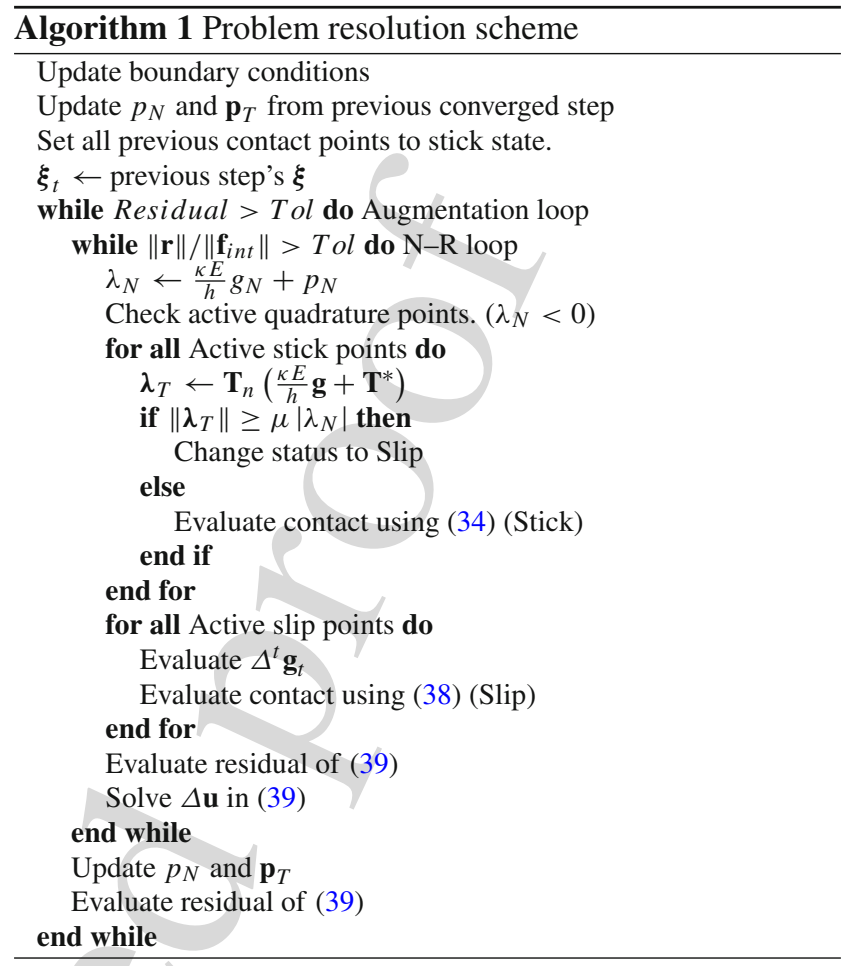

so the computational cost of the solution is not substantially increased.

\subsection{Linearization}

The Newton-Raphson solver needs the linearization of the equations that solve the contact problem. This work will only describe the linearization of $\delta \Pi_{C}$ for both stick and slip cases. The linearization of the contact contribution in the stick case is

$\Delta \delta \Pi_{C_{\text {Stick }}}=\sum_{g}\left[\frac{\kappa E}{h} \Delta \mathbf{g} \cdot \delta \mathbf{g}\right]\left|J_{g}\right| H_{g}$

The definition of the linearization $\Delta \mathbf{g}$ is in this case equivalent to its variation (25), as there is no change of contact coordinates during the stick state. The linearization of the contact contribution in the slip state is shown in (42). For the sake of simplicity, the following definition has been included in the linearization:

$$
\begin{gathered}
\Delta^{t} \mathbf{g}_{t}=\frac{\mathbf{p}_{T}-\frac{\kappa E}{h} \mathbf{T}_{n} \Delta^{t} \mathbf{g}}{\left\|\mathbf{p}_{T}-\frac{\kappa E}{h} \mathbf{T}_{n} \Delta^{t} \mathbf{g}\right\|} \\
\Delta \delta \Pi_{C_{S l i p}}=\sum_{g}\left[\frac{\kappa E}{h} \Delta g_{N} \cdot \delta g_{N}+\left(\frac{\kappa E}{h} g_{N}+p_{N}\right)\right. \\
\quad \times \Delta \delta g_{N}-\mu \frac{\kappa E}{h} \Delta g_{N}\left(\Delta^{t} \mathbf{g}_{t} \cdot \delta \mathbf{g}\right)
\end{gathered}
$$




$$
\begin{aligned}
& -\mu\left(\frac{\kappa E}{h} g_{N}+p_{N}\right)\left(\Delta \Delta^{t} \mathbf{g}_{t} \cdot \delta \mathbf{g}\right) \\
& \left.-\mu\left(\frac{\kappa E}{h} g_{N}+p_{N}\right)\left(\Delta^{t} \mathbf{g}_{t} \cdot \Delta \delta \mathbf{g}\right)\right]\left|J_{g}\right| H_{g}
\end{aligned}
$$

In this case $\Delta g_{N}, \Delta \delta g_{N}, \Delta \Delta^{t} \mathbf{g}_{t}$ and $\Delta \delta \mathbf{g}$ have to be evaluated. As stated in Sect. 3.1, the exact derivative must be calculated for the linearization terms. To evaluate $\Delta g_{N}$ we rearrange (1) and take variations:

$\mathbf{x}^{(2)}\left(\boldsymbol{\xi}^{(2)}\right)=\mathbf{x}^{(1)}+g_{N} \mathbf{n}^{(1)}$

$$
\Delta \mathbf{u}^{(2)}+\frac{\partial \mathbf{x}^{(2)}\left(\xi^{(2)}\right)}{\partial \xi} \Delta \xi+\frac{\partial \mathbf{x}^{(2)}\left(\boldsymbol{\xi}^{(2)}\right)}{\partial \eta} \Delta \eta
$$$$
=\Delta \mathbf{u}^{(1)}+\Delta g_{N} \mathbf{n}^{(1)}+g_{N} \Delta \mathbf{n}^{(1)}
$$

Note that as we are using a ray-tracing scheme to define the contact point pairs, the fixed point is located on the slave body, and the coordinates of the master body are variable. This is contrary to the case of using a closest projection scheme to define the contact point pairs.

As $\mathbf{n}^{(1)}$ is a unit vector, then $\Delta \mathbf{n}^{(1)} \cdot \mathbf{n}^{(1)}=0$ and $\mathbf{n}^{(1)}$. $\mathbf{n}^{(1)}=1$. Therefore, if we multiply (44) by $\mathbf{n}^{(1)}$

$$
\begin{aligned}
& \Delta g_{N}=\left(\Delta \mathbf{u}^{(2)}-\Delta \mathbf{u}^{(1)}\right) \cdot \mathbf{n}^{(1)}+\mathbf{s}_{\xi}^{(2)} \cdot \mathbf{n}^{(1)} \Delta \xi \\
& \quad+\mathbf{s}_{\eta}^{(2)} \cdot \mathbf{n}^{(1)} \Delta \eta
\end{aligned}
$$

where the variables $\Delta \xi$ and $\Delta \eta$ can be calculated solving the linear system of Eqs. (46) resulting from multiplying (44) by vectors $\mathbf{s}_{\xi}^{(1)}$ and $\mathbf{s}_{\eta}^{(1)}$, taking into account that $\mathbf{s}_{\xi}^{(1)} \cdot \mathbf{n}^{(1)}=0$, $\mathbf{s}_{\eta}^{(1)} \cdot \mathbf{n}^{(1)}=0$.

$$
\begin{aligned}
& {\left[\begin{array}{l}
\mathbf{s}_{\xi}^{(2)} \cdot \mathbf{s}_{\xi}^{(1)} \mathbf{s}_{\eta}^{(2)} \cdot \mathbf{s}_{\xi}^{(1)} \\
\mathbf{s}_{\xi}^{(2)} \cdot \mathbf{s}_{\eta}^{(1)} \mathbf{s}_{\eta}^{(2)} \cdot \mathbf{s}_{\eta}^{(1)}
\end{array}\right]\left\{\begin{array}{c}
\Delta \xi \\
\Delta \eta
\end{array}\right\}} \\
& =\left\{\begin{array}{l}
g_{N} \mathbf{s}_{\xi}^{(1)} \cdot \Delta \mathbf{n}^{(1)}-\left(\Delta \mathbf{u}^{(2)}-\Delta \mathbf{u}^{(1)}\right) \cdot \mathbf{s}_{\xi}^{(1)} \\
g_{N} \mathbf{s}_{\eta}^{(1)} \cdot \Delta \mathbf{n}^{(1)}-\left(\Delta \mathbf{u}^{(2)}-\Delta \mathbf{u}^{(1)}\right) \cdot \mathbf{s}_{\eta}^{(1)}
\end{array}\right\}
\end{aligned}
$$

The terms $\Delta \xi, \Delta \eta$ are considered for the calculation of $\Delta \delta g_{N}$ and $\Delta \delta \mathbf{g}$. Therefore, starting from (18) and (25) these increments are respectively written as

$$
\begin{aligned}
\Delta \delta g_{N}= & \left(\delta \mathbf{s}_{\xi}^{(2)} \cdot \mathbf{n}^{(1)}\right) \Delta \xi+\left(\delta \mathbf{s}_{\eta}^{(2)} \cdot \mathbf{n}^{(1)}\right) \Delta \eta \\
& +\left(\delta \mathbf{u}^{(2)}-\delta \mathbf{u}^{(1)}\right) \cdot \Delta \mathbf{n}^{(1)} \\
\Delta \delta \mathbf{g}= & \left(\delta \mathbf{s}_{\xi}^{(2)} \cdot \mathbf{n}^{(1)}\right) \Delta \xi+\left(\delta \mathbf{s}_{\eta}^{(2)} \cdot \mathbf{n}^{(1)}\right) \Delta \eta \\
& +\left(\delta \mathbf{u}^{(2)}-\delta \mathbf{u}^{(1)}\right) \cdot \Delta \mathbf{n}^{(1)}
\end{aligned}
$$

The details of the calculation of $\delta \mathbf{s}_{\xi}^{(2)}, \delta \mathbf{s}_{\eta}^{(2)}$ and $\Delta \mathbf{n}^{(1)}$ and $\Delta^{t} \mathbf{g}_{t}$ are shown in Appendices A and B.

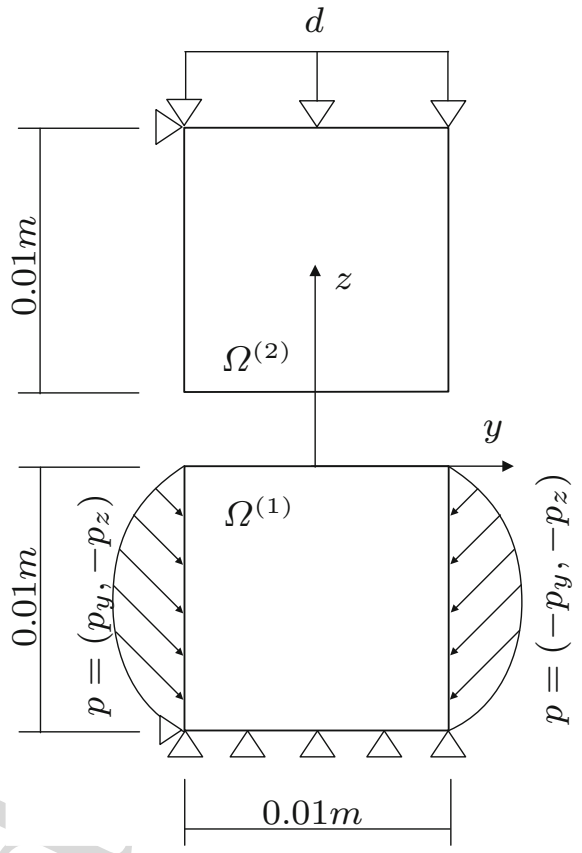

Fig. 5 Example 1. Sketch of the contact problem between two elastic cubes in contact

The linearizations of the Jacobian is also considered, but not shown in this paper as they are standard terms. Its calculation can be easily performed using the tools developed in Appendix A.

\section{Numerical examples}

\subsection{Contact between plane surfaces}

Figure 5 left shows a 2D sketch of the first numerical example, which is the contact simulation between plane surfaces, represented by two elastic cubes. The orientation of the reference system is also shown in the figure, $x$ being the out of plane direction. The separation in the sketch is only for the sake of clarity, as the contact surfaces are overlapping at the initial configuration. A vertical displacement $d=-1.6 \times 10^{-6} \mathrm{~m}$ is applied on the upper face of body 2. The displacements along $y$ direction are constrained on the upper face of body 2 and on the lower face of body 1 . Finally, symmetry conditions are applied to the faces parallel to the $y z$ plane, i.e. this problem can also be analyzed as a plane strain problem. The values of the pressure applied on two lateral faces of body 1 are $p_{y}=4 \times 10^{11}(0.01-z) z P a$ and $p_{z}=10 \times 10^{11}(0.01-z) z P a$. Material properties are common for both bodies, the Young modulus being $E=115 G P a$ and the Poisson coeficient $v=0.3$.

First we will test the convergence of the solution solving a frictionless contact case. Although there is no analytical 

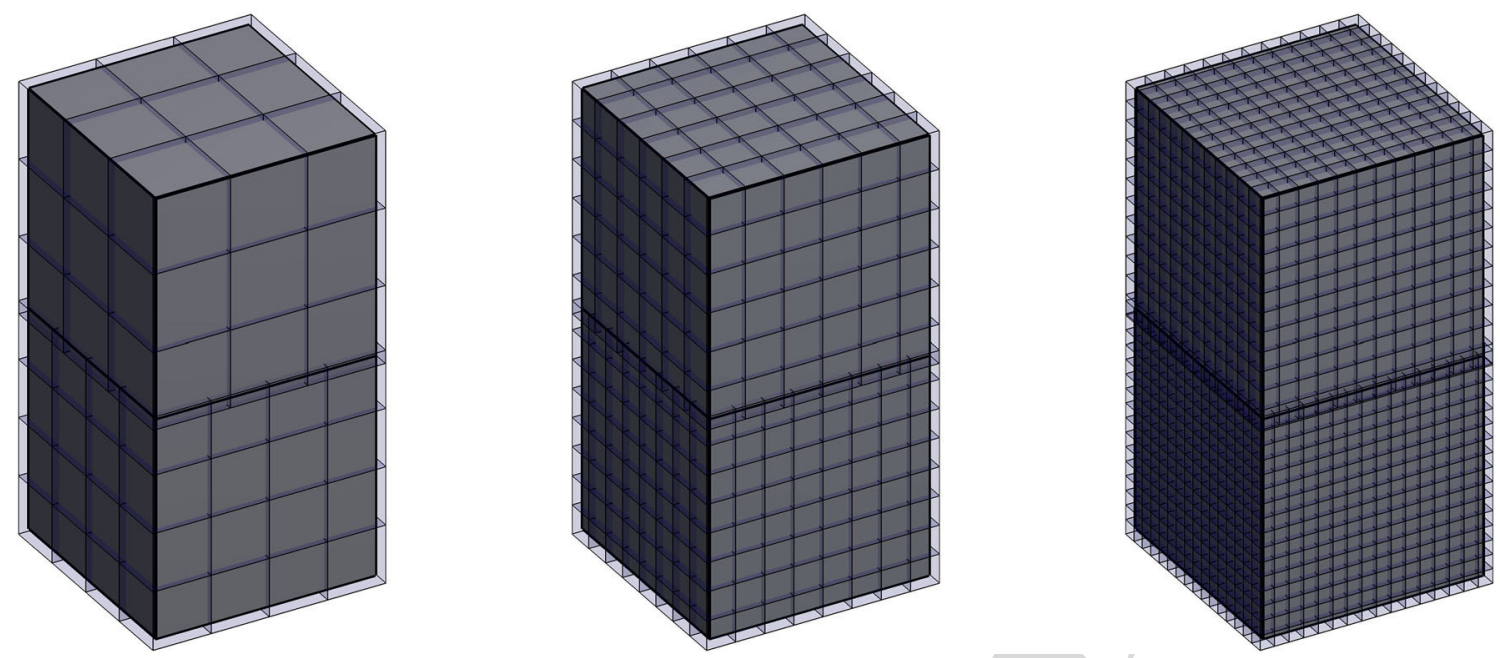

Fig. 6 Example 1. Refinement process for the study of the convergence of the solution. Meshes 1 to 3 are shown from left to right

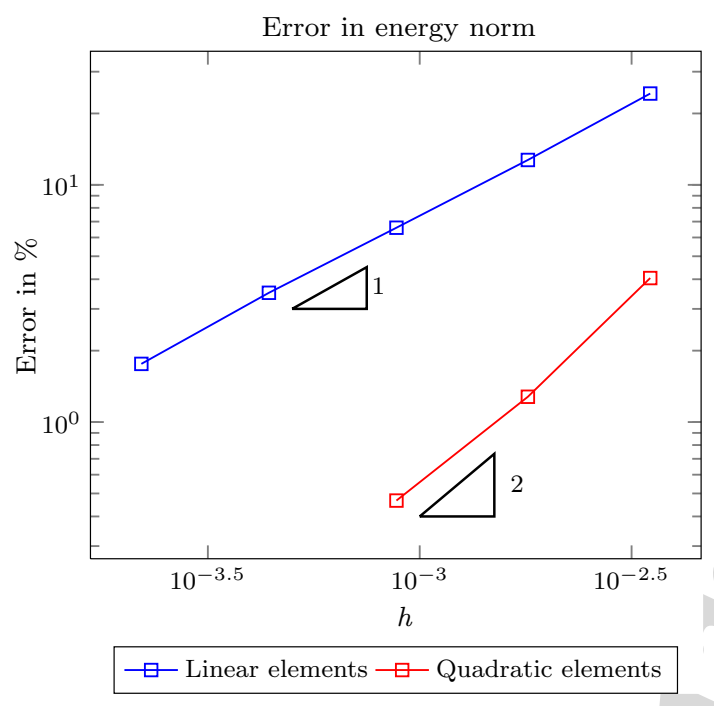

Fig. 7 Example 1: Evolution of the error in the energy norm as a function of the element size for the frictionless contact case. Analysis of the convergence of the solution. The element size is referred to the lower body

solution for this problem, we will use the solution of a $2 \mathrm{D}$ overkill mesh from [42] as a reference to measure the discretization error. Non-conforming Cartesian grids are used on both bodies. Figure 6 shows some of the meshes used for the analysis. The initial mesh consists in a $3 \times 3 \times 3$ grid for the upper body and a $4 \times 4 \times 4$ grid for the lower body. In order to avoid nodes over the boundary for this test, the initial grids are built adding a small offset to the cubes. A set of uniformly $h$-refined meshes is then built by subdividing each element into 8 new elements. Figure 7 shows the relative error in energy norm for a sequence of 5 meshes using linear elements, $\mathscr{H}_{8}$, and 3 meshes using quadratic elements , $\mathscr{H}_{20}$. The results show that the theoretical convergence rate of the error in energy norm, represented by the triangles, is achieved both for $\mathscr{H}_{8}$ and $\mathscr{H}_{20}$ elements.

The recovered contact stress $p_{N}$ is shown in Fig. 8 for the solution of the finest mesh. In this figure, positive values of stresses represent compression. The graph on the right shows the evolution of the contact stress on the $y z$ plane (this profile remains constant along the $x$ direction) for meshes 2 to 5 . The results show that the values of the contact pressure appropriately converge to the reference solution from [42].

Now the same problem is solved considering frictional contact with a friction coefficient $\mu=1.0$. In this case we have used non-conforming manually $h$-adapted meshes for both bodies, as depicted in Fig. 9. Starting with the initial mesh of Fig. 5, we refined the elements over the contact surface multiple times. The surrounding elements were refined as well to keep the difference of the refinement level between adjacent elements below or equal to one.

The results of this problem are shown in Fig. 10. The graph on the left shows the values of the multipliers $\lambda_{N}=$ $p_{N}+\frac{\kappa E}{h} g_{N}$ and $\lambda_{T}=\mathbf{p}_{T}+\frac{\kappa E}{h} \mathbf{g}_{T}$. The blue dashed line represents the values of $-\lambda_{N}$. We can observe the slip and stick areas, with $\left\|\lambda_{T}\right\|=\mu\left|\lambda_{N}\right|$ over the sliding area and $\left\|\lambda_{T}\right\| \leq \mu\left|\lambda_{N}\right|$ over the adhesion area. All these results are similar to those obtained in [42]. The values of the smoothed stress field $p_{N}$ and $\left\|\mathbf{p}_{T}\right\|$ are represented in the graph on the right. This smoothed field is evaluated without taking into account any constraint, hence the differences between the multiplier values. The imposition of the contact constraints to evaluate this smoothed field to get a better solution using the SPR-C technique [37] will be considered in future work.

\subsection{Hollow sphere under internal pressure}

The second example consists of a hollow sphere under internal pressure, which is divided into two independent volumes. 

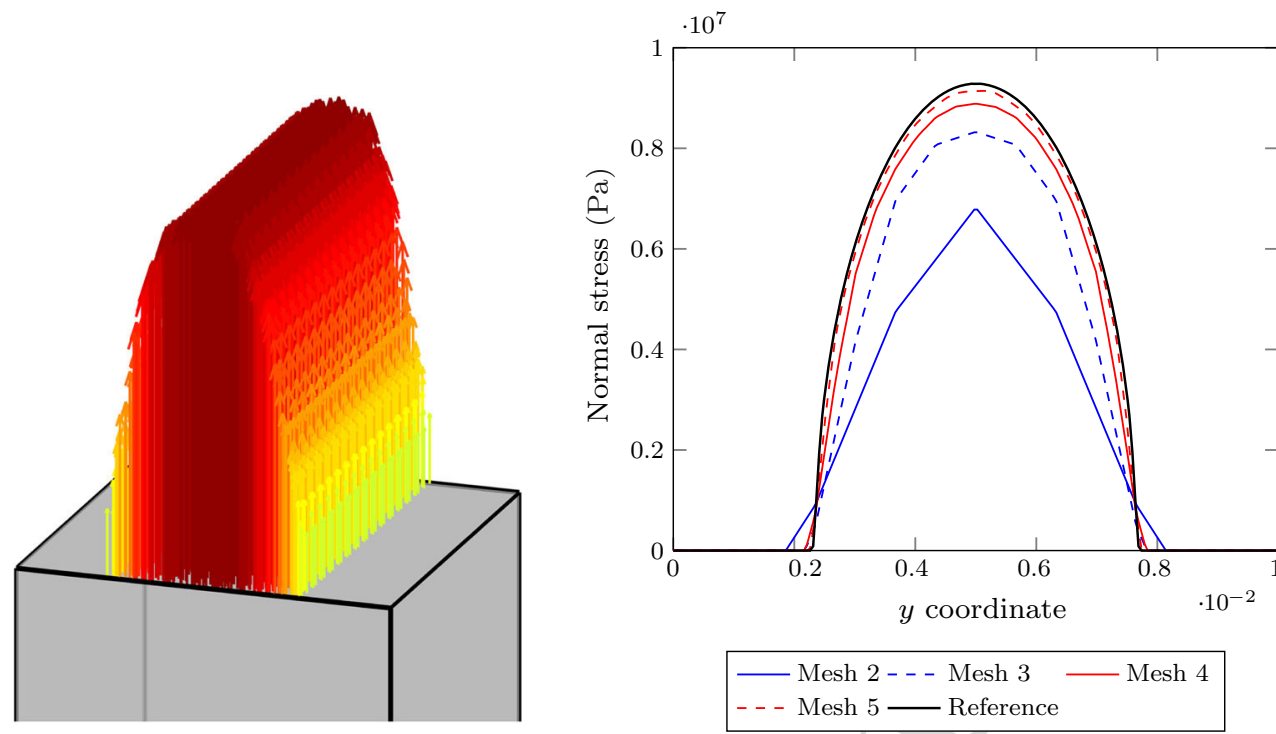

Fig. 8 Example 1. Frictionless contact. Left: Normal stress on the contact area (positive values of the stress stand for compression). Right: Evolution of values of the normal stress, along a path that follows the $y$ direction, with mesh refinement

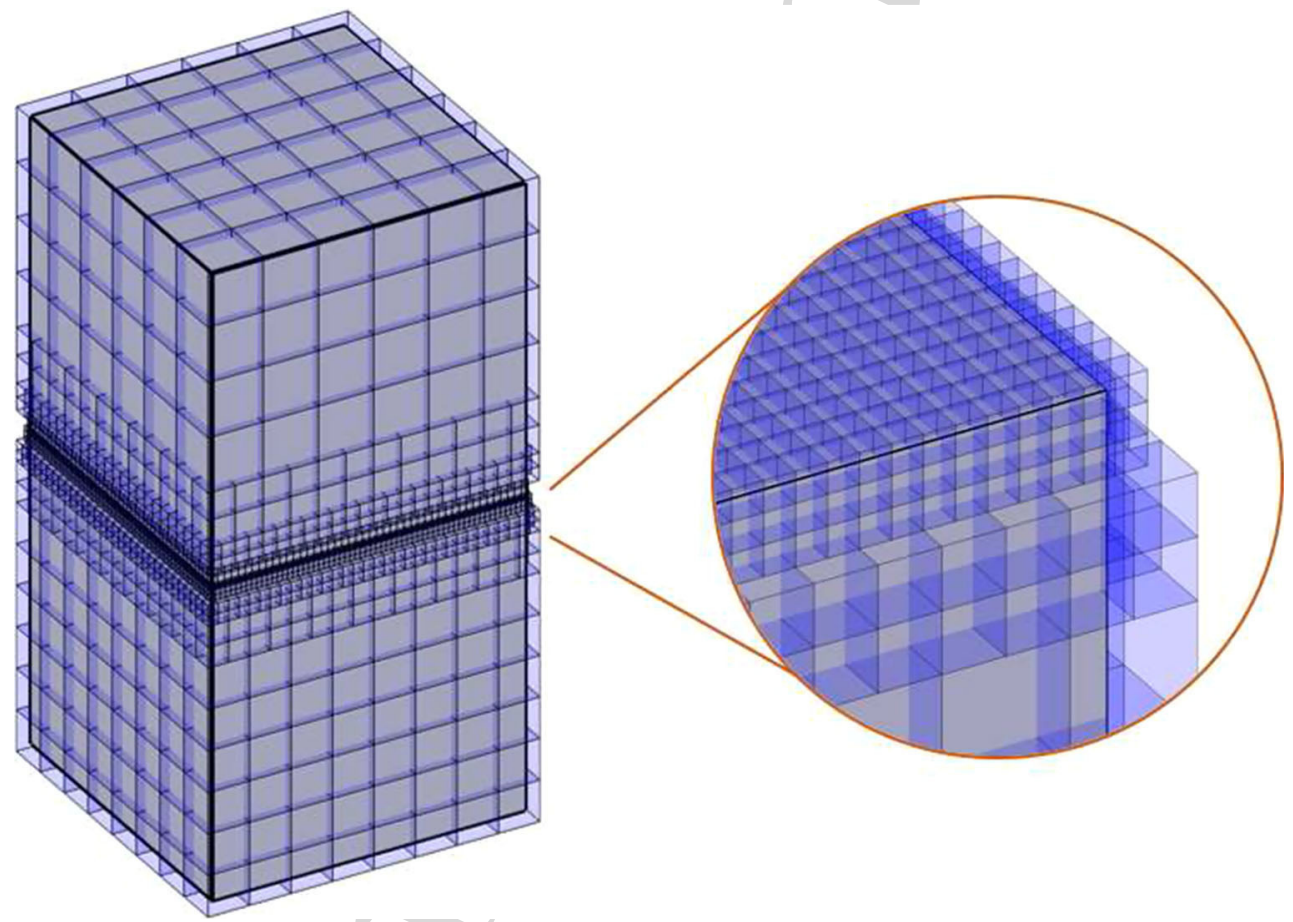

Fig. 9 Example 1. Frictional contact $h$-adapted mesh. The image on the right shows a detail of the refinement of the mesh along the contact surface of the bottom body

In this problem we have curved contact surfaces. We can exactly evaluate the discretization error, as there is an analytical solution. It is easy to express the analytical solution of the problem in spherical coordinates $(r, \theta, \phi)$. The transformation from Cartesian to spherical coordinates is as follows:

$$
\begin{aligned}
r & =\sqrt{x^{2}+y^{2}+z^{2}} \\
\theta & =\arccos \frac{z}{r} \\
\phi & =\arctan \frac{y}{x}
\end{aligned}
$$



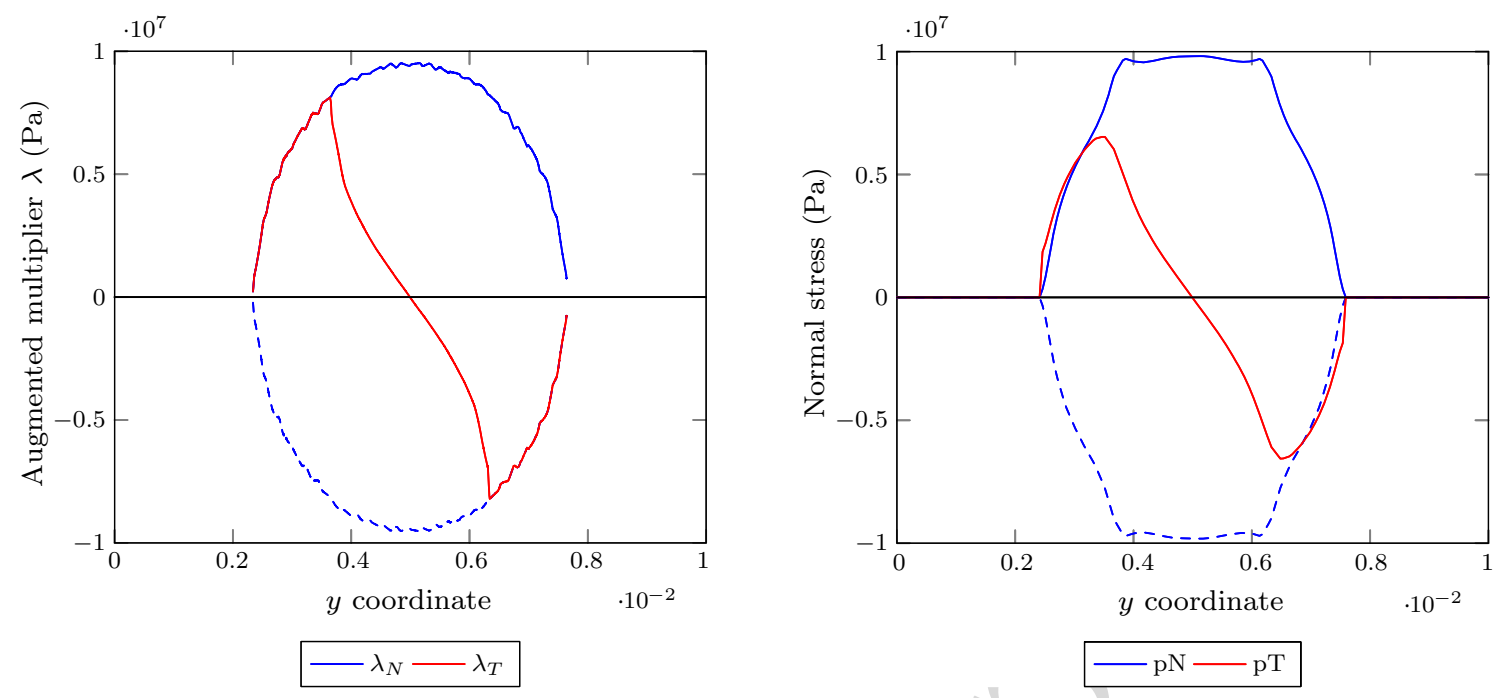

Fig. 10 Example 1. Frictional contact along a path that follows the $y$ direction. Left: values of the augmented Lagrange multipliers. Right: smoothed stress field recovered using SPR
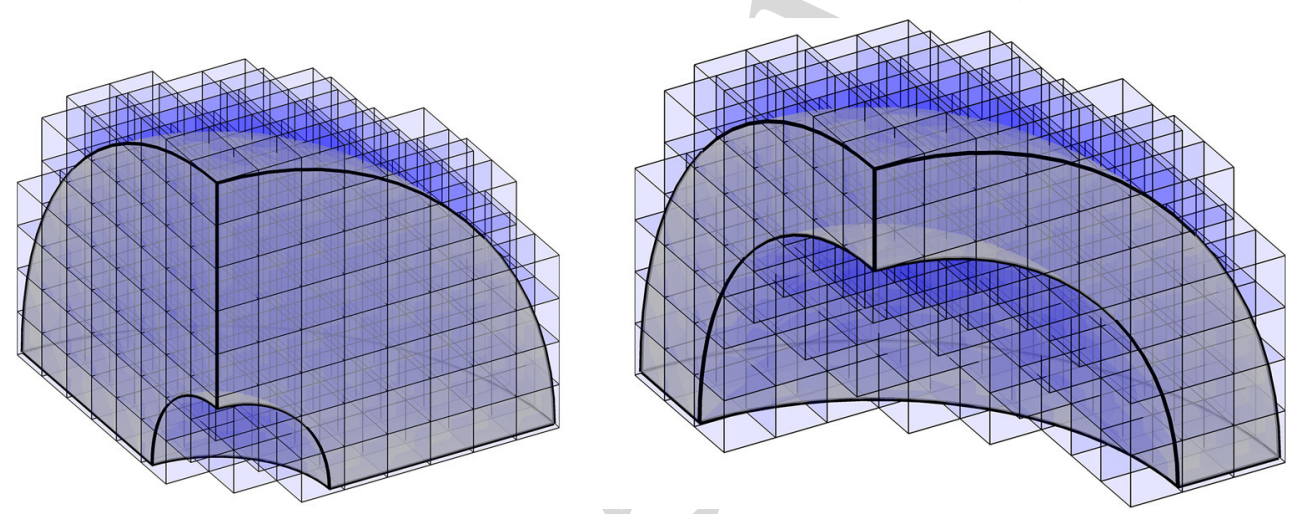

Fig. 11 Example 2. First calculation meshes. The sphere is divided into two volumes, which are discretized using non-conforming Cartesian grids

Then, the analytical stress field corresponding to this problem is:

$$
\sigma_{r}=-P \frac{a^{3}}{b^{3}-a^{3}}\left(\frac{b^{3}}{r^{3}}-1\right)
$$

$$
\sigma_{\theta}=\sigma_{\phi}=P \frac{a^{3}}{b^{3}-a^{3}}\left(\frac{b^{3}}{2 r^{3}}+1\right)
$$

$P$ being the value of the compressive load applied to the internal surface of the sphere, $a$ the inner radius and $b$ the outer radius of the complete hollow sphere. For this example the smaller sphere has an inner radius $a=5$, the outer radius of the bigger sphere is $b=20$ and the contact interface is located at radius $c=15$. One eighth of the hollow spheres with the appropriate symmetry conditions has been used to create the analysis model, as shown in Fig. 11. The material properties chosen for the problem are $E=1000, v=0.3$. The applied internal pressure is $P=1$.

Following the procedure used in the previous example, a series of non-conforming, uniformly $h$-refined meshes were

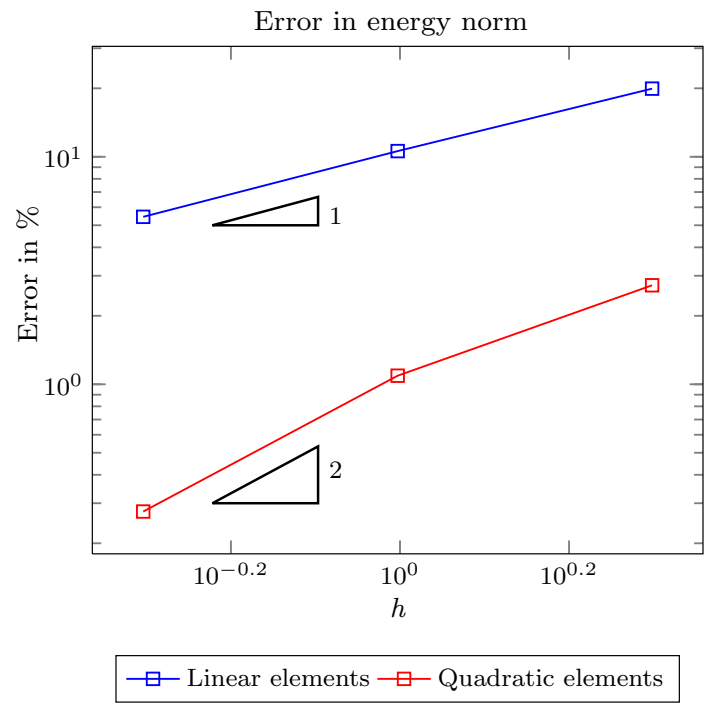

Fig. 12 Example 2. Energy norm error of the solution as a function of the element size. Analysis of the convergence of the solution. The optimal convergence rates are depicted by the triangles below the curves 


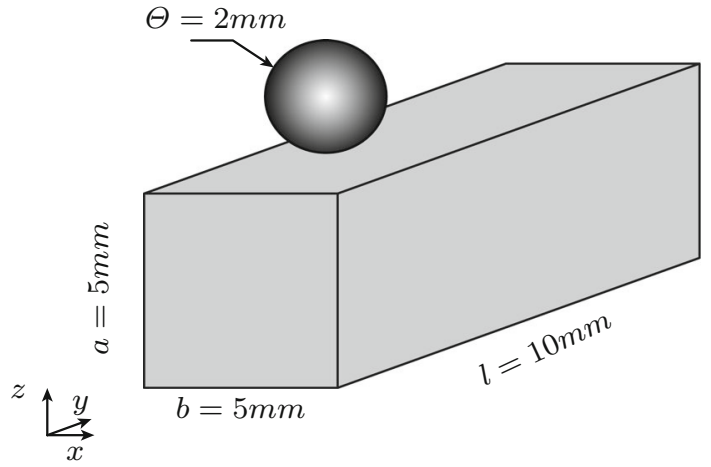

Fig. 13 Example 3. Scheme of the ironing problem

Table 1 Parameters of the ironing problem

\begin{tabular}{lll}
\hline Young modulus of the slab & $E_{\text {Slab }}$ & $100(\mathrm{GPa})$ \\
Poisson coefficient of the slab & $v_{\text {Slab }}$ & 0.3 \\
Young modulus of the sphere & $E_{\text {Sphere }}$ & $1000(\mathrm{GPa})$ \\
Poisson coefficient of the sphere & $v_{\text {Sphere }}$ & 0.3 \\
Vertical displacement of the sphere & $\Delta u_{z}$ & $-0.3(\mathrm{~mm})$ \\
Horizontal displacement of the sphere & $\Delta u_{y}$ & $5(\mathrm{~mm})$ \\
Friction coefficient & $\mu$ & 0.3 \\
\hline
\end{tabular}

solved to test the convergence of the solution. The first calculation mesh is shown in Fig. 11. Figure 12 shows the evolution of the relative exact error in energy norm of the solution with $\mathscr{H}_{8}$ and $\mathscr{H}_{20}$ elements. The optimal convergence rate, depicted by the triangles in the graph, is again achieved for both element types.

\subsection{Frictional contact under large deformations}

The last example in this paper is an ironing problem under large deformations, similar to the ones solved in [42] and [17]. Figure 13 shows the dimensions of the bodies in contact. Material properties and displacements of the problem are shown in Table 1. The ironing block consists of a sphere

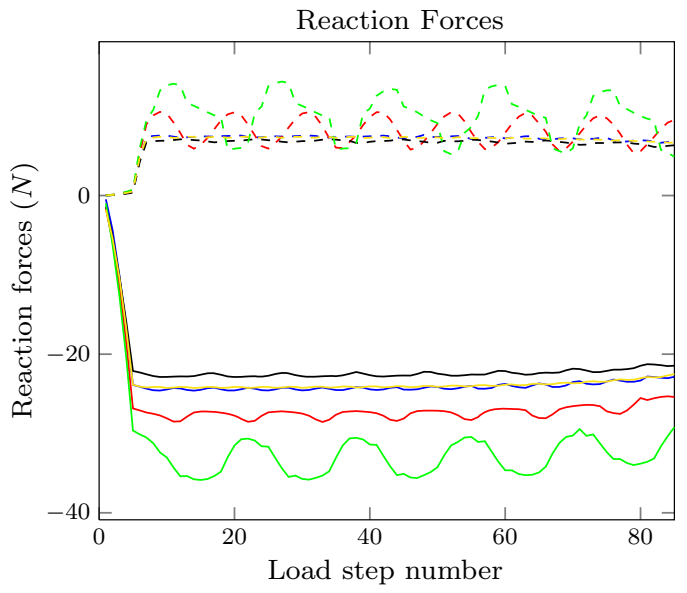

$-F_{z}$, refined $\mathscr{H}_{8}-F_{z}$, uniform $\mathscr{H}_{8}-F_{z}$, uniform $\mathscr{H}_{20}$
$-F_{z}$, ANSYS coarse $-F_{z}$, ANSYS ref. $--F_{y}$, refined $\mathscr{H}_{8}$
$--F_{y}$, uniform $\mathscr{H}_{8}--F_{y}$, uniform $\mathscr{H}_{20}--F_{y}$, ANSYS ${ }^{\circledR}$ coarse
$--F_{y}$, ANSYS ${ }^{\circledR}$ ref.

Fig. 15 Example 3. Reaction forces on the lower face of the block

modelled by four surfaces. The upper surfaces of the sphere are moved towards the slab in 5 time steps, after which a motion along the $y$ direction is applied using 80 time steps. We used a Neo-Hookean material model [45] to consider large deformations of the solids.

This problem was solved with three different meshes. Figure 14 shows the mesh for the first two analyses on the left, with $\mathscr{H}_{8}$ elements for the first analysis and $\mathscr{H}_{20}$ elements for the second. A manual $h$-adaptive refinement was performed on the contact surface of the slab to create the third analysis mesh (Fig. 14 right), using only $\mathscr{H}_{8}$ elements this time. Two different meshes with $\mathscr{H}_{8}$ elements were solved using ANSYS ${ }^{\circledR}[1]$ in order to compare the results. The first of the meshes was created using a discretization similar to the one used in the first mesh in Fig. 14. The second was an overkilled mesh which served as a reference.

Figure 15 shows the sum of the vertical and horizontal reaction forces measured on the lower face of the slab for all

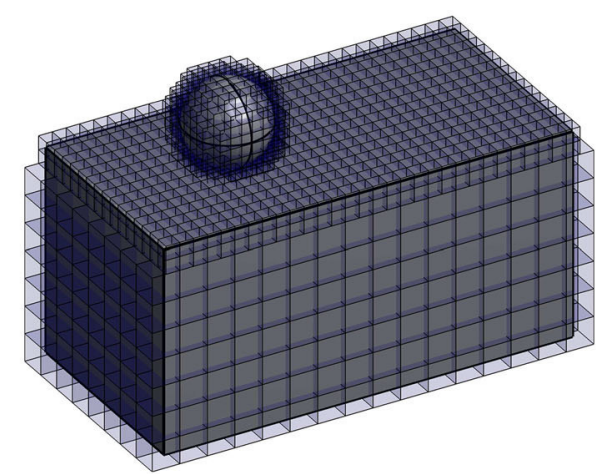

Fig. 14 Example 3. Calculation meshes of the ironing problem. Left: uniform initial meshes. Right: manually adapted mesh on the lower body 

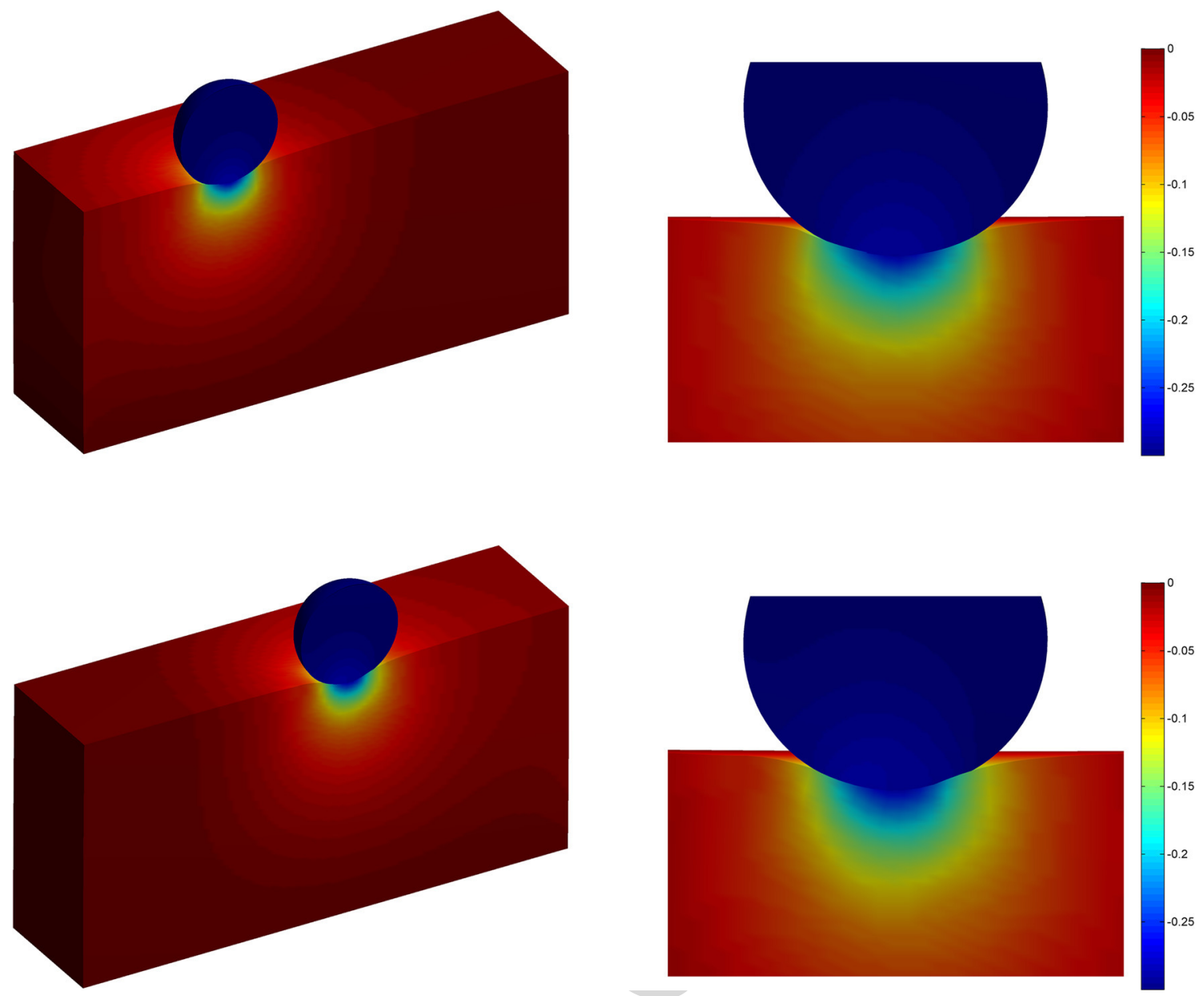

Fig. 16 Example 3. Deformed configuration and vertical displacements $u_{z}$ for the ironing problem for different load steps. On the top, the last load step with only vertical displacement is represented. On the bottom,

results from load step 45. These results correspond to the analysis of a coarse mesh using quadratic $\mathscr{H}_{20}$ elements

the analyses. The results are similar to those obtained with ANSYS ${ }^{\circledR}$, with the values of the reaction forces tending to the reference value with refinement of the mesh. It should be noted that the use of a coarse mesh with $\mathscr{H}_{20}$ elements provides a smooth evolution of the reaction forces, close to the reference values. This is thanks to the definition of the exact geometry, which is independent of the resolution of the mesh. In all cases the wave lengths of the oscillations that appear in the reaction forces are equal to the size of the mesh and are caused by the interaction of the discretized surfaces, which vary with the element size. The deformed configuration for two different load steps is represented in Fig. 16.

\section{Conclusions}

This paper has extended the formulation first proposed in [41] to the case of large deformation frictional contact. In this method a stabilization term that is iteratively computed is added to an augmented Lagrangian formulation, after which the Lagrange multipliers are condensed for the stick and slide case, ensuring a smooth transition between both states.

The formulation was implemented within the three dimensional version of the Cartesian grid Finite Element Method (cgFEM). For this purpose the deformed configuration was defined as a combination of the NURBS surface definition and the finite element displacement field, which allows the exact definition of the boundaries to be taken into account, an important factor in defining the contact kinematics.

Some numerical examples were solved to test the method, using linear 8-node and quadratic 20-node elements. The results show that the appropriate convergence rates are achieved, and the transition between sticking and sliding states is sufficiently smooth. Although the present work may not outperform the more established body-fitted contact formulations in terms of precision or efficiency, it allows solving large sliding contact problems within the embedded domain framework and would be of interest for the solution 
of problems like contact wear simulation, fretting fatigue or prosthesis-tissue interaction.

Acknowledgements The authors wish to thank the Spanish Ministerio de Economia y Competitividad the Generalitat Valenciana and the Universitat Politècnica de València for their financial support received through the projects DPI2013-46317-R, Prometeo 2016/007 and the FPI2015 program.

\section{A Variation of normal and tangent vectors}

We recall here (15) for the calculation of $\delta \mathbf{n}^{(1)}$.

$$
\begin{aligned}
\mathbf{n}^{(1)}= & \frac{\hat{\mathbf{n}}^{(1)}}{\left\|\hat{\mathbf{n}}^{(1)}\right\|} ; \quad \hat{\mathbf{n}}^{(1)}=\mathbf{s}_{\xi}^{(1)} \times \mathbf{s}_{\eta}^{(1)} \\
\delta \mathbf{n}^{(1)}= & \frac{\delta \mathbf{s}_{\xi}^{(1)} \times \mathbf{s}_{\eta}^{(1)}+\mathbf{s}_{\xi}^{(1)} \times \delta \mathbf{s}_{\eta}^{(1)}}{\left\|\hat{\mathbf{n}}^{(1)}\right\|} \\
& -\frac{\mathbf{n}^{(1)}}{\left\|\hat{\mathbf{n}}^{(1)}\right\|}\left[\mathbf{n}^{(1)} \cdot\left(\delta \mathbf{s}_{\xi}^{(1)} \times \mathbf{s}_{\eta}^{(1)}+\mathbf{s}_{\xi}^{(1)} \times \delta \mathbf{s}_{\eta}^{(1)}\right)\right]
\end{aligned}
$$

For the calculation of the variation of the tangent vectors $\mathbf{s}_{\xi}^{(1)}$ and $\mathbf{s}_{\eta}^{(1)}$ we start from (16). We will only describe the calculation of $\delta \mathbf{s}_{\xi}^{(1)}$ as the other term, $\delta \mathbf{s}_{\eta}^{(1)}$, has an identical procedure:

$$
\begin{aligned}
\mathbf{s}_{\xi}^{(1)}= & \frac{\partial \mathbf{x}^{(1)}}{\partial \xi}=\frac{\partial S(\xi, \eta)}{\partial \xi} \\
& +\sum_{j}\left(\frac{\partial N_{j}}{\partial \zeta_{1}^{e}} \frac{\partial \zeta_{1}^{e}}{\partial \xi}+\frac{\partial N_{j}}{\partial \zeta_{2}^{e}} \frac{\partial \zeta_{2}^{e}}{\partial \xi}+\frac{\partial N_{j}}{\partial \zeta_{3}^{e}} \frac{\partial \zeta_{3}^{e}}{\partial \xi}\right) \mathbf{u}_{j}^{(1)}
\end{aligned}
$$

$$
\begin{aligned}
\delta \mathbf{s}_{\xi}^{(1)} & =\delta\left(\frac{\partial \mathbf{x}^{(1)}}{\partial \xi}\right)=\frac{\partial \delta \mathbf{u}^{(1)}}{\partial \xi} \\
& =\sum_{j}\left(\frac{\partial N_{j}}{\partial \zeta_{1}^{e}} \frac{\partial \zeta_{1}^{e}}{\partial \xi}+\frac{\partial N_{j}}{\partial \zeta_{2}^{e}} \frac{\partial \zeta_{2}^{e}}{\partial \xi}+\frac{\partial N_{j}}{\partial \zeta_{3}^{e}} \frac{\partial \zeta_{3}^{e}}{\partial \xi}\right) \delta \mathbf{u}_{j}^{(1)}
\end{aligned}
$$

The linearization of all these variables has the same structure as the variation, so the variations $\delta \mathbf{n}^{(1)}, \delta \mathbf{s}_{\xi}^{(1)}$ and $\delta \mathbf{s}_{\eta}^{(1)}$ can be directly substituted for the increments $\Delta \mathbf{n}^{(1)}, \Delta \mathbf{s}_{\xi}^{(1)}$ and $\Delta \mathbf{s}_{\eta}^{(1)}$.

\section{B Linearization of $\Delta^{t} \mathbf{g}_{t}$}

We recall the definition of $\Delta^{t} \mathbf{g}_{t}$ here:

$$
\Delta^{t} \mathbf{g}_{t}=\frac{\mathbf{p}_{T}-\frac{\kappa E}{h} \mathbf{T}_{n}\left(\mathbf{x}^{(1)}-\mathbf{x}^{(2)}\left(\boldsymbol{\xi}_{t}\right)\right)}{\left\|\mathbf{p}_{T}-\frac{\kappa E}{h} \mathbf{T}_{n}\left(\mathbf{x}^{(1)}-\mathbf{x}^{(2)}\left(\xi_{t}\right)\right)\right\|}
$$

If we use the simplification of (56), the linearization of $\Delta^{t} \mathbf{g}_{t}$ can be expressed as in (57)

$$
\Delta^{t} \mathbf{g}_{t}=\frac{\hat{\mathbf{d}}}{\|\hat{\mathbf{d}}\|} ; \quad \hat{\mathbf{d}}=\mathbf{p}_{T}+\frac{\kappa E}{h} \mathbf{T}_{n}\left(\mathbf{x}^{(2)}\left(\xi_{t}\right)-\mathbf{x}^{(1)}\right)
$$

$\Delta \Delta^{t} \mathbf{g}_{t}=\frac{\Delta \hat{\mathbf{d}}}{\|\hat{\mathbf{d}}\|}-\frac{\Delta^{t} \mathbf{g}_{t}}{\|\hat{\mathbf{d}}\|}\left[\Delta^{t} \mathbf{g}_{t} \cdot \Delta \hat{\mathbf{d}}\right]$

Finally, for the linearization of $\hat{\mathbf{d}}$ we can rearrange Eq. (56) as:

$$
\hat{\mathbf{d}}=\mathbf{p}_{T}+\frac{\kappa E}{h}\left\{\left(\mathbf{x}^{(2)}-\mathbf{x}^{(1)}\right)-\left[\left(\mathbf{x}^{(2)}-\mathbf{x}^{(1)}\right) \cdot \mathbf{n}^{(1)}\right] \mathbf{n}^{(1)}\right\}
$$

With this definition we have a clearer linearization term, which is the following:

$$
\begin{aligned}
\Delta \hat{\mathbf{d}}= & \frac{\kappa E}{h}\left\{\Delta \mathbf{u}-\left[\Delta \mathbf{u} \cdot \mathbf{n}^{(1)}+\left(\mathbf{x}^{(2)}-\mathbf{x}^{(1)}\right) \cdot \Delta \mathbf{n}^{(1)}\right] \mathbf{n}^{(1)}\right. \\
& \left.+\left[\left(\mathbf{x}^{(2)}-\mathbf{x}^{(1)}\right) \cdot \mathbf{n}^{(1)}\right] \Delta \mathbf{n}^{(1)}\right\}
\end{aligned}
$$

where $\Delta \mathbf{u}=\Delta \mathbf{u}^{(2)}\left(\xi_{t}\right)-\Delta \mathbf{u}^{(1)}$. Notice that the local coordinates of the master body are not unknowns, but the coordinates from the last converged step.

\section{References}

1. ANSYS $^{\circledR}$ Academic Research Mechanical, Release 16.2

2. Alart P, Curnier A (1991) A mixed formulation for frictional contact problems prone to Newton like solution methods. Comput Methods Appl Mech Eng 92(3):353-375. https://doi.org/ 10.1016/0045-7825(91)90022-X. http://linkinghub.elsevier.com/ retrieve/pii/004578259190022X

3. Annavarapu C, Hautefeuille M, Dolbow JE (2012) Stable imposition of stiff constraints in explicit dynamics for embedded finite element methods. Int J Numer Methods Eng 92(June):206-228. https://doi.org/10.1002/nme.4343

4. Annavarapu C, Hautefeuille M, Dolbow JE (2014) A Nitsche stabilized finite element method for frictional sliding on embedded interfaces. Part I: single interface. Comput Methods Appl Mech Eng 268:417-436. https://doi.org/10.1016/j.cma.2013.09.002

5. Annavarapu C, Settgast RR, Johnson SM, Fu P, Herbold EB (2015) A weighted nitsche stabilized method for small-sliding contact on frictional surfaces. Comput Methods Appl Mech Eng 283:763-781. https://doi.org/10.1016/j.cma.2014.09.030. www. elsevier.com/locate/cma

6. Baiges J, Codina R, Henke F, Shahmiri S, Wall WA (2012) A symmetric method for weakly imposing Dirichlet boundary conditions in embedded finite element meshes. Int J Numer Methods Eng 90(5):636-658. https://doi.org/10.1002/nme.3339
755 756 
7. Béchet É, Moës N, Wohlmuth B (2009) A stable Lagrange multiplier space for stiff interface conditions within the extended finite element method. Int J Numer Methods Eng 78(8):931-954. https:// doi.org/10.1002/nme. 2515

8. Béchet E, Moës N, Wohlmuth B (2009) A stable Lagrange multiplier space for stiff interface conditions within the extended finite element method. Int J Numer Methods Eng 78:931-954. https:// doi.org/10.1002/nme.2515

9. Belgacem F, Hild P, Laborde P (1998) The mortar finite element method for contact problems. Math Comput Model 28(4-8):263-271. https://doi.org/10.1016/S08957177(98)00121-6. http://www.sciencedirect.com/science/article/ pii/S0895717798001216linkinghub.elsevier.com/retrieve/pii/S08 95717798001216

10. De Lorenzis L, Wriggers P, Zavarise G (2012) A mortar formulation for 3D large deformation contact using NURBS-based isogeometric analysis and the augmented Lagrangian method. Comput Mech 49(1):1-20. https://doi.org/10.1007/s00466-011-0623-4

11. Dittmann M, Franke M, Temizer I, Hesch C (2014) Isogeometric Analysis and thermomechanical Mortar contact problems. Comput Methods Appl Mech Eng 274:192-212. https://doi.org/10.1016/j.cma.2014.02.012. https://ac.elscdn.com/S0045782514000693/1-s2.0-S0045782514000693main.pdf?_tid=a3922af4-a45e-11e7-9f49-00000aab0f01\& acdnat $=1506611392 \_8$ aed5c35cef41733a0dd7c2b5296f8b5

12. Dolbow J, Moës N, Belytschko T (2001) An extended finite element method for modeling crack growth with frictional contact. Comput Methods Appl Mech Eng 190:6825-6846. https://doi.org/10.1016/ S0045-7825(01)00260-2

13. Dolbow JE, Devan a (2004) Enrichment of enhanced assumed strain approximations for representing strong discontinuities: addressing volumetric incompressibility and the discontinuous patch test. Int J Numer Methods Eng 59(1):47-67. https://doi.org/ 10.1002/nme.862

14. Fischer KA, Wriggers P (2006) Mortar based frictional contact formulation for higher order interpolations using the moving friction cone. Comput Methods Appl Mech Eng 195(3740):5020-5036. https://doi.org/10.1016/j.cma.2005.09.025. http://www.sciencedirect.com/science/article/pii/S0045782505005359 linkinghub.elsevier.com/retrieve/pii/S0045782505005359

15. Giovannelli L, Ródenas J, Navarro-Jiménez J, Tur M (2017) Direct medical image-based Finite Element modelling for patient-specific simulation of future implants. Finite Elem Anal Des. https://doi. org/10.1016/j.finel.2017.07.010

16. Gitterle M, Popp A, Gee MW, Wall WA (2010) Finite deformation frictional mortar contact using a semi-smooth Newton method with consistent linearization. Int J Numer Methods Eng. https://doi.org/ $10.1002 / \mathrm{nme} .2907$

17. Hammer ME (2013) Frictional mortar contact for finite deformation problems with synthetic contact kinematics. Comput Mech 51(6):975-998. https://doi.org/10.1007/s00466-012-0780-0

18. Hansbo P, Rashid A, Salomonsson K (2015) Least-squares stabilized augmented Lagrangian multiplier method for elastic contact. Finite Elem Anal Des 116:32-37. https://doi.org/10.1016/j.finel. 2016.03.005

19. Haslinger J, Renard Y (2009) A new fictitious domain approach inspired by the extended finite element method. SIAM J Numer Anal 47(2):1474-1499. https://doi.org/10.1137/070704435

20. Hautefeuille M, Annavarapu C, Dolbow JE (2012) Robust imposition of Dirichlet boundary conditions on embedded surfaces. Int $\mathrm{J}$ Numer Methods Eng 90:40-64. https://doi.org/10.1002/nme.3306

21. Heintz P, Hansbo P (2006) Stabilized Lagrange multiplier methods for bilateral elastic contact with friction. Comput Methods Appl Mech Eng 195(33-36):4323-4333. https://doi.org/10.1016/j. cma.2005.09.008. http://ac.els-cdn.com/S0045782505004238/ 1-s2.0-S0045782505004238-main.pdf?_tid=af348d8a- 25b4-11e7-8608-00000aacb362\&acdnat $=1492684542$ 61b3399a4e2f6ce3876347c69b0c7db7linkinghub.elsevier.com/ retrieve/pii/S0045782505004238

22. Hughes T, Cottrell J, Bazilevs Y (2005) Isogeometric analysis: CAD, finite elements, NURBS, exact geometry and mesh refinement. Comput Methods Appl Mech Eng 194(39-41):4135-4195. https://doi.org/10.1016/j.cma.2004.10.008. http://linkinghub. elsevier.com/retrieve/pii/S0045782504005171

23. Laursen $\mathrm{T}$ (2003) Computational contact and impact mechanics: fundamentals of modelling interfacial phenomena in nonlinear finite element analysis. Springer, Berlin

24. Liu F, Borja RI (2008) A contact algorithm for frictional crack propagation with the extended finite element method. Int J Numer Methods Eng 76(June):1489-1512. https://doi.org/10.1002/nme. 2376

25. Liu F, Borja RI (2010) Stabilized low-order finite elements for frictional contact with the extended finite element method. Comput Methods Appl Mech Eng 199(37-40):2456-2471. https://doi.org/ 10.1016/j.cma.2010.03.030

26. Marco O, Sevilla R, Zhang Y, Ródenas JJ, Tur M (2015) Exact 3D boundary representation in finite element analysis based on Cartesian grids independent of the geometry. Int J Numer Methods Eng 103(6):445-468. https://doi.org/10.1002/nme.4914

27. Nadal E, Ródenas JJ, Albelda J, Tur M, Tarancón JE, Fuenmayor FJ (2013) Efficient finite element methodology based on cartesian grids: application to structural shape optimization. Abstr Appl Anal 2013:1-19. https://doi.org/10.1155/2013/ 953786. http://www.hindawi.com/journals/aaa/2013/953786/

28. Neto D, Oliveira M, Menezes L, Alves J (2016) A contact smoothing method for arbitrary surface meshes using nagata patches. Comput Methods Appl Mech Eng 299:283-315. https://doi.org/10. 1016/j.cma.2015.11.011. http://www.sciencedirect.com/science/ article/pii/S0045782515003643

29. Nistor I, Guiton MLE, Massin P, Moës N, Géniaut S (2009) An $\mathrm{X}$-FEM approach for large sliding contact along discontinuities. Int J Numer Methods Eng 78:1407-1435. https://doi.org/10.1002/ nme. 2532

30. Oliver J, Hartmann S, Cante JC, Weyler R, Hernández JA (2009) A contact domain method for large deformation frictional contact problems. Part 1: theoretical basis. Comput Methods Appl Mech Eng 198:2591-2606. https://doi.org/10.1016/j.cma.2009.03.006. https://ac.els-cdn. $\mathrm{com} / \mathrm{S} 004578250900125 \mathrm{X} / 1$-s2.0-S004578250900125Xmain.pdf?_tid=fefda67e-a9dc-11e7-b8d3-00000aacb361\& acdnat $=1507215409 \_8107$ ea7818dd7c4d2799bf8d9df98d06

31. Piegl L, Tiller W (1995) The NURBS Book. Springer, Berlin

32. Pietrzak G, Curnier A (1999) Large deformation frictional contact mechanics: continuum formulation and augmented Lagrangian treatment. Comput Methods Appl Mech Eng 177(3-4):351381. https://doi.org/10.1016/S0045-7825(98)00388-0. http:// linkinghub.elsevier.com/retrieve/pii/S0045782598003880

33. Poulios K, Renard Y (2015) An unconstrained integral approximation of large sliding frictional contact between deformable solids. Comput Struct 153:75-90. https://doi.org/10.1016/j.compstruc. 2015.02.027

34. Puso MA, Laursen TA (2004) A mortar segment-to-segment frictional contact method for large deformations. Comput Methods Appl Mech Eng 193(45-47):4891-4913. https://doi.org/10.1016/ j.cma.2004.06.001

35. Renard Y (2013) Generalized Newton's methods for the approximation and resolution of frictional contact problems in elasticity. Comput Methods Appl Mech Eng 256:38-55. https://doi.org/10. 1016/j.cma.2012.12.008

36. Ribeaucourt R, Baietto-Dubourg MC, Gravouil A (2007) A new fatigue frictional contact crack propagation model with the cou- 
pled X-FEM/LATIN method. Comput Methods Appl Mech Eng 196:3230-3247. https://doi.org/10.1016/j.cma.2007.03.004

37. Ródenas JJ, Tur M, Fuenmayor FJ, Vercher A (2007) Improvement of the superconvergent patch recovery technique by the use of constraint equations: The SPR-C technique. Int J Numer Methods Eng 70:705-727. https://doi.org/10.1002/nme.1903

38. Rogers DF (2001) An introduction to NURBS: with historical perspective. Elsevier, Amsterdam

39. Temizer I, Wriggers P, Hughes TJR (2012) Three-dimensional mortar-based frictional contact treatment in isogeometric analysis with NURBS. Comput Methods Appl Mech Eng 209-212:115128. https://doi.org/10.1016/j.cma.2011.10.014

40. Tur M, Albelda J, Marco O, Ródenas JJ (2015) Stabilized method of imposing Dirichlet boundary conditions using a recovered stress field. Comput Methods Appl Mech Eng 296:352-375. https://doi. org/10.1016/j.cma.2015.08.001

41. Tur M, Albelda J, Navarro-Jimenez JM, Rodenas JJ (2015) A modified perturbed Lagrangian formulation for contact problems. Comput Mech. https://doi.org/10.1007/s00466-015-1133-6

42. Tur M, Fuenmayor FJ, Wriggers $P$ (2009) A mortar-based frictional contact formulation for large deformations using Lagrange multipliers. Comput Methods Appl Mech Eng 198(37-40):2860-2873. https://doi.org/10.1016/j.cma.2009.04.007
43. Tur M, Giner E, Fuenmayor F, Wriggers P (2012) 2d contact smooth formulation based on the mortar method. Comput Methods Appl Mech Eng 247-248:1-14. 10.1016/j.cma.2012.08.002.http:// www.sciencedirect.com/science/article/pii/S0045782512002381

44. Wriggers P (2006) Computational contact mechanics. Springer, Berlin

45. Wriggers $P$ (2008) Nonlinear finite element methods. Springer, Berlin. https://doi.org/10.1007/978-3-540-71001-1. http:// scholar.google.com/scholar?hl=en\&btnG=Search\&q=intitle: No+Title\#0\%5Cnhttp://link.springer.com/content/pdf/10.1007/ 978-3-540-71001-1.pdfhttp://link.springer.com/10.1007/978-3540-71001-1http://www.ncbi.nlm.nih.gov/pubmed/15369193

46. Yang B, Laursen TA, Meng X (2005) Two dimensional mortar contact methods for large deformation frictional sliding. Int $\mathrm{J}$ Numer Methods Eng 62(9):1183-1225. https://doi.org/10.1002/nme.1222

47. Zienkiewicz OC, Zhu JZ (1992) The superconvergent patch recovery and a posteriori error estimates. Part 1: the recovery technique. Int J Numer Methods. https://doi.org/10.1002/nme.1620330702

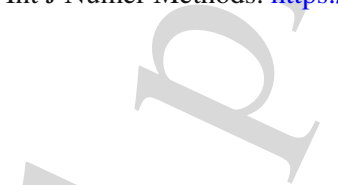

